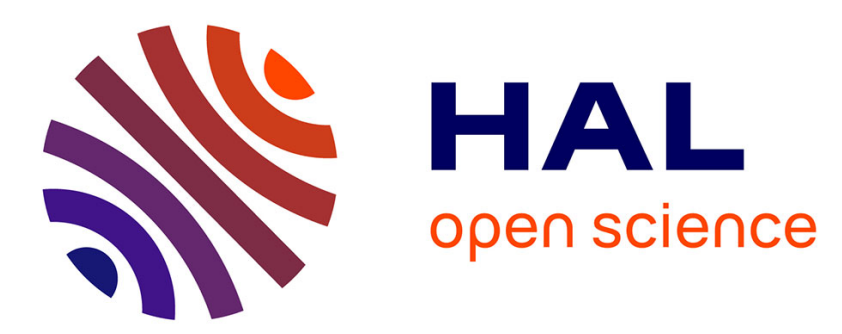

\title{
Convection activity over the Guinean coast and Central Africa during northern spring from synoptic to intra-seasonal timescales
}

Pierre Honoré Kamsu Tamo, Serge Janicot, D. Monkam, André Lenouo

\section{- To cite this version:}

Pierre Honoré Kamsu Tamo, Serge Janicot, D. Monkam, André Lenouo. Convection activity over the Guinean coast and Central Africa during northern spring from synoptic to intra-seasonal timescales. Climate Dynamics, 2014, 43 (12), pp.3377-3401. 10.1007/s00382-014-2111-y · hal-01102836

\section{HAL Id: hal-01102836 https: / hal.sorbonne-universite.fr/hal-01102836}

Submitted on 13 Jan 2015

HAL is a multi-disciplinary open access archive for the deposit and dissemination of scientific research documents, whether they are published or not. The documents may come from teaching and research institutions in France or abroad, or from public or private research centers.
L'archive ouverte pluridisciplinaire HAL, est destinée au dépôt et à la diffusion de documents scientifiques de niveau recherche, publiés ou non, émanant des établissements d'enseignement et de recherche français ou étrangers, des laboratoires publics ou privés. 
Convection activity over the Guinean coast and Central Africa during northern spring from synoptic to intra-seasonal timescales

\author{
P.H. Kamsu-Tamo ${ }^{1}$, S. Janicot ${ }^{1}$, D. Monkam ${ }^{2}$ and A. Lenouo ${ }^{2}$ \\ ${ }^{1}$ Sorbonne Universités (UPMC, Univ Paris 06)-CNRS-IRD-MNHN, LOCEAN laboratory, 4 \\ Place Jussieu, F-75005 Paris, France \\ ${ }^{2}$ University of Douala, LPA, BP24157, Douala, Cameroon
}

\begin{abstract}
This study proposes an overview of the main synoptic, medium-range and intraseasonal modes of convection and precipitation in northern spring (March-June 1979-2010) over West and Central Africa, and to understand their atmospheric dynamics. It is based on daily NOAA OLR and CLAUS Tb convection data, daily TRMM and GPCP rainfall products and daily ERA-Interim reanalysis atmospheric fields. It is first shown that mesoscale convective systems can be modulated in terms of occurrences number and intensity at such time scales. Based on EOF analyses on the 2-90-day filtered data it is shown that the main mode of convective and rainfall variability is located along the Guinean coast with a moderate to weak extension over Central Africa. Corresponding regressed deseasonalised atmospheric fields highlight an eastward propagation of patterns consistent with convectively coupled equatorial Kelvin wave dynamics. Then a Singular Spectrum Analysis combined with a Hierarchical Ascendant Classification enable to define objectively the main spectral bands of variability within the 2-90-day band, and highlight three main bands, 2-8-day, 8-22-day and 20-90-day. Within these three bands, space-time spectral decomposition is used to identify the relative impacts of Convectively Coupled Equatorial Kelvin, Rossby and Inertia-Gravity waves, as well as MJO signal. It confirms that eastward propagating signals (Convectively Coupled Equatorial Kelvin wave and MJO) are highly dominant in these convection and precipitation variability modes over the Guinean coast during northern spring. So, while rain-producing individual systems are moving westward, their activity are highly modulated by sub-regional and regional scales envelops moving to the east. This is a burning issue for operational forecasting centers to be able to monitor and predict such eastward propagating envelops of convective activity.
\end{abstract}

Key words: Synoptic variability; Intra-seasonal variability ; African monsoon ; Convectively coupled equatorial wave. 


\section{Introduction}

The AMMA ${ }^{1}$ international program on the understanding of the West African monsoon and its impacts has been the opportunity to highlight the evidence of intraseasonal variability of convective activity and rainfall over this region during northern summer and to investigate its related mechanisms. One synthesis of these results has been published in Janicot et al. (2011). Three main modes of variability have been identified, two of them with a mean medium-range periodicity of 15 days and another one with a mean intra-seasonal periodicity around 40 days. Both have a regional extension and represent an envelope modulating the convective activity of individual mesoscale convective systems. These modes are intermittent but their impact on precipitation and convective activity can be strong when they occur. They have also a marked zonal propagation character.

(i) The 40-day mode modulates convection over the whole West and East Africa domain and propagates westward, closely associated to the Madden-Julian Oscillation (MJO) signal over the Indian sector. Its westward part is mainly controlled by convectively coupled equatorial Rossby (CCER) waves (Matthews 2004, Janicot et al. 2009, Lavender and Matthews 2009, Pohl et al. 2009, Janicot et al. 2010, Ventrice et al. 2011, Mohino et al. 2012). (ii) The 15-day Sahelian mode propagates westward too, modulating convection mostly over the Sahelian band, and is linked mainly to CCER waves (Janicot et al. 2010) and to midlatitude medium-range variability through a major role played by the Saharan heat low (Chauvin et al. 2010, Roehrig et al. 2011). (iii) The Quasi-Biweekly Zonal Mode (QBZD; Mounier et al. 2008) modulates convection over the Guinean Coast, combining an eastward propagating signal from the Atlantic to East Africa associated to some convectively coupled equatorial Kelvin (CCEK) wave signal, with a 4-day stationary phase over the Guinean Coast. All these modes appear to be controlled both by internal atmospheric dynamics and land-surface interactions (Mounier et al. 2008, Taylor 2008, Lavender et al 2009). They can also have some impact on the African summer monsoon onset characterized by an abrupt northward shift of the Inter-Tropical Convergence Zone (ITCZ) at the end of June (Sultan and Janicot 2003, Mounier et al. 2008, Janicot et al. 2008). (iv) At a shorter synoptic timescale it has been also shown that westward propagating African easterly waves are not the alone synoptic system but that eastward propagating CCEK waves are also present (between 4 and 6-day periodicity), less frequently that easterly waves but with a similar impact on convection and rainfall modulation when they occur (Mounier et al. 2007, Mekonnen et al. 2008, Ventrice et al. 2012a,b, 2013).

Synoptic to intraseasonal variability must be a priori strong during the other seasons of the year when the ITCZ is close to or over the equator, favouring interactions between equatorial atmospheric dynamics and convection. However only few studies have been presently carried out over West and Central Africa outside of northern summer, and none of those that have addressed this issue has provided an overview.

(i) At synoptic timescale Nguyen and Duvel (2008) revealed large oscillations of convective activity with periods of 3 to 6 days over equatorial Africa. In March and April, when the ITCZ migrates northward and crosses the equator, this periodic behaviour is highly pronounced with a marked peak at 5-6 days. Robust horizontal and vertical patterns are consistent with CCEK waves even if this cannot explain all of the periodic behaviour observed over equatorial Africa.

(ii) At medium-range timescale, de Coëtlogon et al. (2010), then Leduc-Leballeur et al. (2011, 2013), performed local diagnostic analyses over the gulf of Guinea and the Guinean Coast

\footnotetext{
${ }^{1}$ African Monsoon Multidisciplinary Analyses ; http.www.amma-international.org
} 
in northern spring and showed the existence of peaks around 15 days in surface winds and sea surface temperatures (SST). They showed through lagged cross-correlations the signature of a 5day lag wind forcing and 3-day lag strong negative SST feedback. A cold SST anomaly covering the equatorial and coastal upwelling is forced after about one week by stronger-than-usual southeasterlies linked to the St Helena anticyclone. Within about $5^{\circ} \mathrm{S}$ and $5^{\circ} \mathrm{N}$, two retroactions between SST and surface wind appear to dominate near-surface atmosphere conditions. When the wind leads the SST, stronger monsoonal winds north of $2^{\circ} \mathrm{N}$ are partly sustained by the developing SST anomaly and bring more humidity and rainfall toward the continent. When the SST leads the wind, a reversal of anomalous winds is observed mainly south of $2^{\circ} \mathrm{N}$, closing a negative feedback loop with a biweekly periodicity. The equatorial SST cooling intensifies a surface-wind equatorial divergence/coastal convergence circulation and generates a cross-equatorial pressure gradient, which both strengthen the southerlies north of the equator. This increases subsidence above the ocean and convection in the northern Gulf of Guinea. Maloney and Shaman (2008), and Nguyen and Duvel (2008), also detected a significant spectral peak at timescale near 15 days but they did not investigate it further more.

(iii) At intra-seasonal timescale, Gu and Adler (2004) showed by a 2D wavelet analysis the dominance of eastward propagating synoptic and intra-seasonal wave signals in rainfall along the Guinean coast in May-June but did not detail their spatial structures. The MJO signal over West Africa and the Atlantic during spring was more detailed by Gu (2008) and Maloney and Shaman (2008), and appears as clear eastward propagating coherent convection and circulation features with maximum amplitude near the gulf of Guinea and in the Atlantic ITCZ, suggesting that the regional intra-seasonal convective signals might be mostly a regional response to the MJO and probably contribute to the MJO's global propagation. More recently Yu et al. (2012) assessed the effects of MJO and CCER waves on surface winds and convection of the tropical Atlantic and African monsoon area. They showed that in general, the MJO events dominate the westwardpropagating CCER waves in affecting strong convection in the African monsoon region. The CCER waves, however, have larger contributions to convection in the western Atlantic basin. Both the westward and eastward propagating signals contribute approximately equally in the central Atlantic basin. Both convection amplitude and the number of strong convective events associated with the MJO are larger during November-April than during May-October. Convection associated with CCER wave events is stronger during November-April, and the numbers of CCER wave events are higher during November-April than during May-October in the African monsoon region, and are comparable for the two seasons in the western and central Atlantic basins. Finally Tchakoutio Sandjon et al. (2012) focused on the MJO signal over Central Africa. They identified three eastward propagating modes over northern Congo, southern Ethiopia and southwestern Tanzania respectively, as well as a strong interannual modulation of MJO over eastern central Africa partially linked with the ENSO events.

The objective of the work presented here is to provide an overview of the main synoptic, medium-range and intra-seasonal modes of convection and precipitation in northern spring (March-June) over West and Central Africa, and to compare their relative weights. This will be useful as a basis for more detailed further studies, in particular to discriminate the role of local surface-atmosphere interactions from remote forcings in this modes dynamics and to evaluate their predictability. The datasets are detailed in section 2. In section 3 we present characteristic examples of synoptic and intra-seasonal sequences of convection and rainfall amounts. In section 4 a reference regional index will be defined, based on Empirical Orthogonal Function (EOF) analyses of 2-90-day filtered convection and precipitation data. We will regress deseasonalised atmospheric fields onto this index to get a first view of the dominant atmospheric patterns and dynamics. Then this index will be decomposed through a Singular Spectrum Analysis (SSA), and a Hierarchical Ascendant Classification (HAC) will be applied on the regression fields associated 
to the SSA components in order to define objectively the main spectral bands of variability within the 2-90-day band. We will highlight three main bands, 2-8-day, 8-22-day and 20-90-day, with a dominance of eastward propagation. In section 5 the Wheeler-Kiladis (1999) space-time spectral analysis will enable to identify the associated CCEK wave dynamics over West and Central Africa. Eastward propagating CCEK signals in the three periodicity bands will be detailed in section 6 and westward propagating CCEK signals in section 7, confirming the dominance of eastward CCEK dynamics. Conclusions will be given in section 8 .

\section{Data}

In this study we have used different products of precipitation and convective activity in order to get robust results, as well as reanalysed atmospheric fields for documenting the spatial patterns associated to convection in the different periodicity bands. The period 1979-2010 will be used as the reference period available for both NOAA OLR et ERAI data (see below), and the other products, available on shorter periods, will help to complement and validate the results.

\subsection{The daily NOAA OLR product}

Since 1974, polar-orbiting National Oceanic and Atmospheric Administration (NOAA) Television and Infrared Observation Satellite (TIROS) satellites have established a quasi-complete series of twice-daily outgoing longwave radiation (OLR) at the top of the atmosphere at a resolution of 2.58 latitude-longitude (Grueber and Krueger 1974). The daily-interpolated OLR dataset produced by the Climate Diagnostic Center (Liebmann and Smith 1996) has been used here over the period of 1979-2010 as a proxy for deep convection. Local hours of the measurements varied between 0230 and 0730 UTC in the morning and between 1430 and 1930 UTC in the afternoon. Because deep convection over West Africa has a strong diurnal cycle, a sample of daily OLR based on two values separated by $12 \mathrm{~h}$ is obtained to get a daily average. Wheeler et al. (2000), Straub and Kiladis (2002), and Roundy and Frank (2004), among others, have illustrated the utility of OLR in tracing convectively coupled equatorial waves. This product will be used as the reference dataset.

\subsection{The daily CLAUS Tb data}

Satellite-observed infrared brightness temperatures $(\mathrm{Tb})$ from the Cloud Archive User Service (CLAUS; Hodges et al. 2000) have also been used over the period 1984-2005 to detect the convective cloudiness at higher spatiotemporal resolution $\left(0.5^{\circ} \times 0.5^{\circ}, 3 \mathrm{~h}\right)$. Daily averages have been computed. This dataset is built from multiple geostationary and polar-orbiting satellite imagery in the infrared window channel. It has been successfully applied to the study of the diurnal cycle by Yang and Slingo (2001) and to the study of African easterly and CCEK waves and convection by Mekonnen et al. $(2006,2008)$.

\subsection{The daily GPCP rainfall product}

The Global Precipitation Climatology Project (GPCP) One-Degree-Daily (1DD) combination has been used over the period of 1997-2008 to complement the results obtained with the other infrared datasets. This rainfall estimate product is based on 3-hourly merged global infrared brightness temperature histograms on a $1^{\circ} \times 1^{\circ}$ grid in the $40^{\circ} \mathrm{N}-40^{\circ} \mathrm{S}$ band (Huffman et al. 2001). It has the advantage of providing global coverage with a better sampling than the NOAA OLR dataset. On the other hand, because it is only available since 1997, it can be used only as a complement to carry out our investigation. Moreover, because it is based on infrared histograms, 
its rainfall estimate may have large errors when rainfall is produced by warm-top clouds.

\subsection{The daily TRMM rainfall product}

In addition, the daily TRMM $3 \mathrm{~B} 42$ rainfall product on a $0.25^{\circ} \mathrm{x} 0.25^{\circ}$ grid has been used over the period 1998-2010. It mixes satellite measurements including the TMI and TRMM precipitation radar to calibrate infrared precipitation estimate from geostationary satellites as well as data from ground radars (Huffman et al., 2007), which guarantees the best possible quality for precipitation data. It is retrieved from the website trmm.gsfc.nasa.gov.

\subsection{The ERA-Interim reanalysis data}

The ERA-Interim reanalysis from the European Centre for Medium- Range Weather Forecasts (ECMWF; hereafter ERAI; Dee et al., 2011) has been used over the period 1979-2010 to document the spatial patterns associated with the convection signals. These reanalyses data are available on a $0.75^{\circ} \times 0.75^{\circ}$ horizontal grid with vertical atmospheric profiles retrieved on 23 levels from 1000 to $100 \mathrm{hPa}$. For this study, the daily mean of the 6-hourly parameters have been computed. Daily OLR and rainfall data are also shown in order to evaluate them in front of the satellite-observed products.

\section{Examples of synoptic and intraseasonal time sequences over the Guinea coast}

Figure 1 presents the mean bi-monthly fields of TRMM rainfall and NOAA OLR from January-February to November-December, as well as the corresponding time-latitude crosssections over West and Central Africa. These panels show the well-known meridional evolution of the ITCZ with a higher spatial resolution for the TRMM product. In particular high rainfall amounts in the Cameroon highlands and west of the Fouta Jalon mountains are well detected over most of the year. From March to June, convection and precipitation are high along the Guinean coast due to the location of the ITCZ over the highest SSTs in the year in the equatorial basin. This precedes the well-known northward shift of the ITCZ over West Africa at the end of June (see Fig.1b left panel) indicating the start of the summer monsoon (Sultan and Janicot 2003). Over Central Africa such an abrupt northward shift does not exist and the ITCZ moves less to the north but summer rainfall starts to increase at the same time (Fig.1b right panel). Similar patterns are given by the other OLR and rainfall products but with lower values for ERAI (not shown).

Figure 2 shows characteristic synoptic, medium-range and intra-seasonal time sequences of convection (CLAUS Tb) and precipitation (TRMM) over the Atlantic-Africa domain in northern spring. These sequences have been selected based on the full investigation presented in the next sections. It is shown here as an illustration and is analysed further in sections 6 and 7. Left panels show (i) the time-longitude sequence of $2.5^{\circ} \mathrm{S}-7.5^{\circ} \mathrm{N}$ averaged $\mathrm{Tb}$ from 1 to 30 March 1999 where the synoptic timescale signal is well defined, (ii) the corresponding daily mean $2.5^{\circ} \mathrm{S}-7.5^{\circ} \mathrm{N} / 5^{\circ} \mathrm{W}$ $5^{\circ} \mathrm{E}$ TRMM rainfall evolution smoothed by a moving-sum over 2 days, and (iii) the corresponding wavelet diagram of the NOAA OLR index computed as an average over $2.5^{\circ} \mathrm{S}-7.5^{\circ} \mathrm{N} / 5^{\circ} \mathrm{W}-5^{\circ} \mathrm{E}$. Middle panels show the sequence from 25 March to 5 May 2001 where the medium-range timescale signal is well defined and daily rainfall evolution is smoothed by a moving-sum over 5 days. The right panels show the sequence from 1 March to 1 July 2003 where the intra-seasonal timescale signal is well defined and daily rainfall evolution is smoothed by a moving-sum over 10 days. All three time-longitude cross-sections show westward propagating signals of mesoscale convective systems ( $\mathrm{Tb}$ shaded in blue) with succession of sequences of more or less convective activity. The impact of these fluctuations is very clear over the Guinean coast in the respective 
daily rainfall time series where high variations with periodicities of about 6 days (synoptic), 15 days (medium-range) and 50 days (intra-seasonal) are highlighted. They are also well detected in

\section{Detection of the main periodicities}

In this section we aim at detecting and identify the main mode of variability in terms of convection and precipitation over West and Central Africa. So a reference regional index based on the first EOF component of 2-90-day filtered convection and precipitation data will be defined. Deseasonalised atmospheric fields will be regressed onto this index to get a first view of the dominant atmospheric patterns and dynamics. Then this index will be decomposed through SSA, and a HAC will be applied on the regression fields associated to the SSA components in order to define objectively the main spectral bands of variability within the 2-90-day band.

\section{$\underline{4.1 \text { Reference index definition }}$}

First eigenvectors of March-June 2-90-day filtered NOAA OLR, CLAUS Tb, ERAI OLR, and GPCP, TRMM and ERAI rainfall have been computed over their respective period (see section 2) over the domain $50^{\circ} \mathrm{W}-50^{\circ} \mathrm{E} / 15^{\circ} \mathrm{S}-20^{\circ} \mathrm{N}$ using the covariance matrix. The variance fields of the reconstruction by these first principal components are shown on Figure 3 with the respective percentages of explained 2-90-day variance by these components. Note that the color scales are different in order to better see the details within each field. All the patterns are consistent and show a strong pole centred over the Guinean coast, including an extension of weaker values over West and Central Africa but for OLR and Tb only. That means that over Central Africa in particular the OLR variance is not due to rainfall variability but to nonprecipitating clouds or atmospheric moisture. The rainfall fields highlight another pole along the equator off the Brasilian coast that is associated with an opposite sign in the eigenvector fields (not shown), meaning that more rainfall over the Guinea gulf is linked to less rainfall to the west. Such dipoles are also present in the OLR eigenvectors but with very weak variances in the western pole (not displayed). Explained variance percentages of these first EOF components are included between $6.7 \%$ and $9.6 \%$ for OLR/Tb, and between $2.7 \%$ and $4.7 \%$ for rainfall, probably due to the higher spatial resolution of the rainfall datasets, to higher small scale variance for rainfall fields and to a highly skewed positive distribution. On Fig.3 while the ERAI OLR variance field shows values of the same order as for NOAA OLR, ERAI rainfall variances are very weak compared to TRMM and GPCP values, meaning a high under-estimation in this reanalysis product. Same EOF analysis performed separately on March-April and May-June show similar patterns but a bit more zonally elongated in May-June (not shown). Based on these results, we can define a reference daily index as the average of 2-90-day filtered NOAA OLR over the area $2.5^{\circ} \mathrm{S}-7.5^{\circ} \mathrm{N} / 5^{\circ} \mathrm{W}-5^{\circ} \mathrm{E}$. This index is correlated at +0.94 to the similar deseasonalised index. Table 1 (first row) shows the correlations between this index and similar indices computed from the five other OLR and rainfall variables. Correlations are higher than 0.7 in absolute value for CLAUS Tb, GPCP and ERAI OLR, which are the products the most similar to NOAA OLR, and between 0.4 and 0.5 in absolute value for TRMM and ERAI rainfall. We have checked that using a similar index computed on a bit different area sizes does not modify the results in the rest of the study. Moreover in order to better evaluate the space representativity of this reference index, we have computed the correlation coefficients between the reference index and indices computed over two larger longitude domains, a first one over $10^{\circ} \mathrm{W}-10^{\circ} \mathrm{E}$ and the second one over $10^{\circ} \mathrm{W}-30^{\circ} \mathrm{E}$. For the largest one, the correlations with the reference indices are equal to $0.82,0.78,0.75,0.77,0.64$ and 0.65 for NOAA-OLR, CLAUS Tb, ERAI OLR, GPCP, TRMM and ERAI rain respectively, meaning a good coherency between West and Central Africa for irradiances and a moderate one for 
precipitation, in consistency with the EOF reconstructed variance patterns. So the reference index defined over the longitude band $5^{\circ} \mathrm{W}-5^{\circ} \mathrm{E}$ is representative of a more extended area including Central Africa.

To evaluate the representativeness of this reference index on the full atmospheric fields and to get a first outlook of the associated atmospheric dynamics, March-June regression patterns of deseasonalised atmospheric variables onto this standardised 2-90-day filtered reference index have been computed, first at time To (with no time lag; Fig.4a), second through time-longitude crosssections from To-20 days to To +20 days (Fig.4b). Regression coefficients are displayed for NOAA OLR, CLAUS Tb, ERA OLR (W.m ${ }^{-2}$ ) with superimposed $925 \mathrm{hPa}$ wind and geopotential height, and for GPCP, TRMM and ERAI rainfall $\left(\mathrm{mm}\right.$. day $\left.^{-1}\right)$ with superimposed $200 \mathrm{hPa}$ divergent wind and velocity potential. These values can be interpreted in terms of their modulation associated to a variation of the NOAA OLR reference index of one standard deviation. The spatial patterns at To are consistent with the EOF eigenvectors and show a high modulation of deseasonalised convection and rainfall centered over the Guinean coast and the equatorial Guinean gulf, with a zonal extension from $30^{\circ} \mathrm{W}$ to $30^{\circ} \mathrm{E}$ for OLR but a weaker one restricted over the oceanic basin for rainfall (with weak values for ERAI rainfall). In particular Central Africa is more weakly affected in terms of precipitation than in terms of OLR, meaning a modulation of non precipitating clouds and/or atmospheric water vapour content. The area of enhanced rainfall and convective activity is associated with a clear upper levels divergent outflow and in the lower levels with east-west pressure gradient and enhanced easterly wind components in quadrature with the OLR peak, that is characteristic of a Kelvin wave pattern. This is confirmed by the timelongitude cross-sections of Fig.4b that highlight eastward propagations of both the convective envelop and the associated pressure-wind patterns. This signal is high from $60^{\circ} \mathrm{W}$ to $90^{\circ} \mathrm{E}$ but with weaker modulations over Central Africa. This enhanced convective signal is preceded and followed by an opposite modulation but no clear periodicity appears at this stage. One can notice again the very weak values of ERAI rainfall compared to TRMM and GPCP products. On Fig.4a a zonal band of negative values is also present along $30^{\circ} \mathrm{N}$ over northern Africa and Saudi Arabia in NOAA and ERAI OLR but not in rainfall fields, meaning atmospheric moisture or nonprecipitating clouds probably associated with tropical plumes (Knippertz and Fink 2009). Regressed atmospheric patterns onto the other irradiance and rainfall indices averaged over the same reference area have also been computed and they have very similar patterns (not shown).

327

328

329

330

331

332

333

334

335

336

337

338

339

340

341

342

343

344

\subsection{Spectral analysis of the reference index}

To better identify the main modes of variability within the 2-90-day band, spectral analysis and decomposition have been carried out based on the reference index. Figure 5 shows the MarchJune smoothed spectra of the NOAA OLR reference index and of the other indices computed over the same area $2.5^{\circ} \mathrm{S}-7.5^{\circ} \mathrm{N} / 5^{\circ} \mathrm{W}-5^{\circ} \mathrm{E}$ (CLAUS Tb, ERAI OLR, and GPCP, TRMM and ERAI rainfall) on their respective periods (see section 2). Significant peaks ( $95 \%$ of the red noise spectrum) are detected on the NOAA OLR reference index mainly between 30 and 60 days, around 25 days and 15 days, and between 5 and 10 days. These main periodicities are also present and mostly significant for the other five indices.

To go further into this spectral decomposition and to identify the main periodicity bands, a SSA has been performed on the NOAA OLR reference index. Such a procedure was used in Janicot et al. (2010). SSA (Vautard and Ghil 1989; Vautard et al. 1992; Ghil et al. 2002) is related to EOF analysis but is applied to a lagged time series providing SSA modes that correspond to oscillations in a specific frequency band. It is well designed to extract periodic information from noisy time series. Given the time series of the reference index as $\mathbf{x}(\mathbf{t})$ of length $\mathbf{N}, \mathbf{x}$ is embedded 
in a vector space of dimension $\mathbf{M}$ to represent the behaviour of the system by a succession of overlapping views of the series through a sliding M-point window. SSA provides eigenvectors, the EOFs in the time domain (T-EOFs), and quasi-periodic modes appear as pairs of degenerate eigenvalues associated with T-EOFs in quadrature. The projection of the original time series onto the $\mathbf{k}^{\text {th }}$ T-EOF gives the corresponding principal components in the time domain (T-PCs). One can reconstruct the part of the original time series associated with the mode $\mathbf{k}, \mathrm{RC}_{\mathbf{k}}$, by combining the $\mathbf{k}^{\text {th }}$ T-EOF and the $\mathbf{k}^{\text {th }}$ T-PC. The RCs are additive and the original time series can be reconstructed by summing up the $\mathbf{M}$ reconstructed components $\mathrm{RC}_{\mathbf{k}}$. The choice of the window size $\mathrm{M}$ is arbitrary. It must be large enough to get as much information as possible and yet small enough to ensure many repetitions of the original signal by maximizing the ratio N/M (Ghil et al. 2002). A value of 60 has been used for $\mathbf{M}$ to accommodate the intra-seasonal signal at 40-60-day. Similar analyses have been performed for different values of $\mathbf{M}$ from 40 to 60 , and the results were not very sensitive to these different window sizes.

The results of the SSA applied on the 2-90-day filtered reference index are presented in Table 2 and Figure 6. Tab.2 shows the explained variance of the first 30 T-EOFs $(84 \%$ for their sum). Oscillatory modes can be detected by pairs of eigenvalues that are approximately equal and by T-EOFs in quadrature. Fig.6 displays the smoothed spectra of the 2-90-day filtered NOAA OLR reference index (same as Fig.5) and of the reconstructed signals by pairs of components $\mathrm{RC}_{1-}$ ${ }_{2}$ to $\mathrm{RC}_{29-30}$. The first oscillatory mode is captured by the first pair of T-EOFs, representing $12.2 \%$ of the variance and is characterized by a periodicity band between 30 and 60 days with higher energy near 50 days. The following pair of T-EOFs represents another oscillatory mode $(9.0 \%$ of the variance) with high energy between 20 and 30 days and a spectral peak around 25 days. The third pair of T-EOFs extracts a bit weaker oscillatory mode $(7.8 \%$ of variance) with the highest energy between 12.5 and 16.5 days and a spectral peak at 15 days, and the fourth pair (7.1\%) between 16.5 and 22 days with a peak around 18 days. Other T-EOFs pairs exhibit shorter periodicities from 12.5 days to 3.5 days. On Fig. 6 one can see that not all the variance is retrieved by SSA but what is retrieved and highlighted by SSA corresponds to periodic signals.

Our aim is then to classify these RC modes in order to define objectively the main periodicity bands. The first step has been to compute maps of deseasonalised OLR over the domain $15^{\circ} \mathrm{N}-15^{\circ} \mathrm{S} / 180^{\circ} \mathrm{W}-180^{\circ} \mathrm{E}$ regressed onto each of the $15 \mathrm{RC}$ modes $\left(\mathrm{RC}_{1-2}\right.$ to $\left.\mathrm{RC}_{29-30}\right)$, as was done in Fig.4. The regression maps at To highlight similar spatial patterns with progressive shorter zonal wavelength as the RC periodicities are decreasing (not shown). All of them show eastward propagative signals between To-20 days and To+20 days, consistent with Kelvin-type signals (not shown). The second step is to aggregate these spatial To patterns into a smaller number of types based on their similarities. For that we apply the HAC using a distance between every two maps based on the correlation coefficient: Dist $=2 \times(1-C)$ where $C$ is the correlation between two of these regression coefficients maps. This enables to quantify all these distances in terms of spatial similarity independently of the amplitude of the regression coefficients, and to classify them into different classes using the Ward metric (Saporta 1990). The resulting dendrogram is shown in Figure 7. A test is applied at each significant cutting level of the dendogram defined by the "rule of the elbow", that is the level where there is a significant change of the aggregation index, based on this metric. We chose first to select a classification into four classes, just before a drop of this index when one goes from four to five classes. This discriminates the $\mathrm{RC}_{1-2}$ to $\mathrm{RC}_{3-4}$ (20-90-day periodicity band), the $\mathrm{RC}_{5-6}$ to $\mathrm{RC}_{13-14}$ (8-22-day periodicity band), the $\mathrm{RC}_{15-16}$ to $\mathrm{RC}_{25-26}$ (the 5-8-day periodicity band) and the $\mathrm{RC}_{27-28}$ to $\mathrm{RC}_{29-30}$ (periodicities below 5 days). As the $\mathrm{RC}_{27-28}$ to $\mathrm{RC}_{29-30}$ variance is very weak and periods short and close to 5 days, we finally chose to aggregate $\mathrm{RC}_{15-16}$ to $\mathrm{RC}_{29-30}$ and include also shorter periodicities. Then SSA and HAC enable to discriminate three periodicity bands, 20-90-day, 8-22-day and 2-8-day, what will 
be used in the following sections. The SSA decomposition has produced a continuum of spatial regression patterns with progressively shorter wavelengths with decreasing periodicities, leading to classes with a good internal consistency, and then to a weak loss of variability when applying HAC. Sensitivity tests on the boundaries of these three bands have been carried out that provide similar results in the rest of the study (not shown). Another point is that our results would not have be different if we had used the other products than NOAA OLR to define the boundaries of the components but on their classification and related boundaries of spectral bands that is sensitive. We have checked that the other dendrograms provide similar results (not shown).

Tab.1 (second row) shows the correlations of the RCPs reconstructed NOAA OLR index over the 20-90-day band and of the other 20-90-day band filtered indices with the 20-90-day NOAA OLR reference index, as well as similar correlations for the 8-22-day band (third row) and the 8-22-day band (fourth row). All of these coefficients are significant at least at 5\%, and more for most of them. It confirms that the three RCPs reconstructed NOAA OLR signals represent very well the corresponding three bands variability, and that, as seen in first column of Tab.1, the other OLR products have also high correlations, as for rain products except ERAI. These correlations are globally weaker for the 2-8-day band (fourth row), due to the fact that the considered RCPs do not extract periodicities between 2 and 4 days (see Fig.6). Finally one can notice that the 2-8-day, 8-22-day and 20-90-day filtered indices are correlated at $+0.64,+0.53$, +0.47 respectively with the deseasonalised reference index. EOF analysis similar to Fig. 3 but for each of the three periodicity bands have been performed and shows a first eigenvector with high weights still located along the Guinean coast confirming that our reference index is also relevant at these timescales (not shown). This will be detailed in section 6 .

\section{Spectral space-time analysis}

We have seen that the 2-90-day filtered OLR main mode, as well as pairs of RC modes, have a spatial pattern and an eastward propagation consistent with CCEK signals. Our aim is to investigate more precisely this dynamics for the three identified periodicity bands by using the Wheeler-Kiladis (1999) spectral space-time decomposition in order to evaluate in particular the effect of the equatorial dynamics of CCEK, CCER and MJO.

A wavenumber-frequency spectral analysis has been performed on the OLR component symmetric about the equator between $15^{\circ} \mathrm{N}$ and $15^{\circ}$ for February-July $1979-2010$, as well as for the other OLR and rain products over their respective available periods. It has been carried out both on all the longitudes and on restricted longitude domains by tapering data to zero outside of these domains to control spectral leakage. Two domains have been tested: $90^{\circ} \mathrm{W}-60^{\circ} \mathrm{E}$ and $60^{\circ} \mathrm{W}$ $30^{\circ} \mathrm{E}$. The wavenumber-frequency spectral analysis provides similar results for the three domains except that we get stronger signals on restricted domains especially for Kelvin and Westward Inertia-Gravity (WIG) waves (not shown). Figure 8 a shows these results for the intermediate domain $90^{\circ} \mathrm{W}-60^{\circ} \mathrm{E}$. The shaded spectral peaks lie above the $95 \%$ level of significance, and a family of equivalent depth curves for Kelvin, Equatorial Rossby, and IG waves from equatorial linear shallow-water theory (Matsuno 1966) are also shown (see Wheeler and Kiladis 1999 for more details).

The spectra reveal the existence of peaks corresponding to CCEK and CCER waves. The MJO peak is also visible in the spectrum but does not correspond to a shallow-water mode. In Wheeler and Kiladis (1999) a so-called tropical depression band, representing easterly waves for 
Africa, was also identified during northern summer in the westward-propagating signal domain for 2-6-day periods and 6-20 westward zonal wavenumbers. However this signal is no more present in northern winter and is replaced by some signal in the WIG domain centred around periodicity 2.5 days and zonal wavenumber 5 (see Fig.5 of Wheeler and Kiladis 1999). In Fig.8a there is a similar WIG signal and no evidence of an easterly wave signal, what is consistent with the period of the year, February to July, used to compute Fig.8a spectra. The CCEK NOAA OLR signal has evident near 3 days. We can also notice weak amplitudes around 7 days and around 20 days that corresponds well to the boundaries of the periodicity bands identified in section 4 . The main CCER peak is centred around 25 days and the MJO eastward signal appears at periods above 30 days. CLAUS Tb and ERAI OLR show similar peaks but with clearly lower values for ERAI data. These peaks are also present in the rainfall data with again lower values for ERAI.

In Fig. $8 \mathrm{~b}$ the boxes outline the regions of filtering for the CCEK and CCER waves examined here as well as for the WIG waves and the MJO signal. This filtering has been performed by creating an OLR dataset through an inverse transform that retains only the Fourier coefficients corresponding to the designated boxes (Wheeler and Kiladis 1999). Note that the datasets obtained contain equatorial waves as well as a significant amount of background convection. This technique has been applied successfully for the West African summer monsoon in Mounier et al. $(2007,2008)$ and Janicot et al. $(2009,2010)$. Red dots represent the 15 RCs modes computed in section 4 with size proportional to their variance. Their central period was provided from their spectrum and their wavelength estimated from the related regression pattern at To (section 4). The first two modes are well located within the MJO box. Within the Kelvin domain the $3^{\text {rd }}$ to $7^{\text {th }}$ modes between 8 and 22 days and the remaining 8 modes between 4 and 8 days are mostly located along the $25 \mathrm{~m}$ equivalent depth meaning an average phase speed of about $16 \mathrm{~m} . \mathrm{s}^{-1}$, some others along $12 \mathrm{~m}$ (phase speed of $11 \mathrm{~m} . \mathrm{s}^{-1}$ ) and remaining few near $50 \mathrm{~m}$ (phase speed of $22 \mathrm{~m} \cdot \mathrm{s}^{-1}$ ).

\section{Eastward propagation signals}

In this section the spatial patterns associated to the three periodicity bands and the related filtered CCEK and MJO signals are examined. Let us recall that the SSA-HCA approach was used to define objectively the boundaries of the three main spectral bands that are then used for filtering data. Figure 9 revisits the characteristic examples of synoptic, medium-range and intra-seasonal signals shown on Fig. 2 by including superimposed filtered signals. Fig.9a highlights the 2-8-day filtered NOAA OLR signal (red contours) and the 2.5-8-day CCEK filtered NOAA OLR signal (black contours). When the Kelvin signal is high (5-23 March), it is associated with high eastward propagating 2-8-day filtered signal and it modulates very clearly the activity of westward propagating mesoscale convective systems. Outside of this active Kelvin sequence, the 2-8-day filtered signal appears less well organised. Fig.9b highlights the 8-22-day filtered NOAA OLR signal (red contours) and the 8-22-day CCEK filtered NOAA OLR signal (black contours). The 822-day signals appears well organised over the whole sequence and modulates clearly the activity of westward propagating mesoscale convective systems at this timescale. It is characterised by an eastward propagation combined with a systematic stationary phase along the Guinean coast between $15^{\circ} \mathrm{W}$ and $0^{\circ} \mathrm{W}$. The associated Kelvin filtered signal is high during the first half of the sequence only. Fig.9c highlights the 20-90-day filtered NOAA OLR signal (red contours) and the MJO filtered NOAA OLR signal (black contours). Again the activity of westward propagating mesoscale convective systems are modulated at this longer timescale through eastward propagating signals associated most of the time with MJO signals. In consequence these examples show that the westward propagating rain producing systems can be modulated significantly by 
eastward propagative waves at various time and space scales, contributing to define envelop of convective activity, and synoptic to intra-seasonal fluctuations of rain at sub-regional to regional scales.

Figure 10 shows maps of March-June 1979-2010 NOAA OLR variance for filtered 2-8day, 8-22-day and 20-90-day signals, and for superimposed filtered 2.5-8-day CCEK, 8-22-day CCEK and MJO signals respectively (Fig.10 left). Variance percentages of 2-8-day, 8-22-day and 20-90-day signals refered to 2-90-day variance are also shown (Fig.10 right). The synoptic part (28 -day) represents the highest fraction of variance with up to $70 \%-80 \%$ of $2-90$-day variance over West and Central Africa. It is also very high over the rest of the equatorial band except the eastern coast of Africa. The maxima are centred a bit north of the equator over Africa and the East Pacific basin, and a bit south of the equator over the Indian and West Pacific basins as well as South America. The associated Kelvin contribution is high and its maxima are coincident with 2-8-day maxima. The spatial distribution of TRMM rainfall variance is similar to NOAA OLR except weak values over the African continent as seen in Fig.3 (not shown). The variance field of the 822-day signal shows again maxima within the equatorial band with high values over the Indian and Pacific oceans. Other maxima are located along the Guinean coast, along $10^{\circ} \mathrm{N}$ over Africa, and over the western Sahara corresponding here to frequent occurrences of tropical plumes. In this sector the TRMM variance field shows again high values over the Guinean gulf only (not shown). Relative contributions to the 2-90-day NOAA OLR signal are rather of the same order all along the equatorial band about $20-30 \%$ with a bit weaker values over land. Over the Guinea gulf it coincides with the maxima of the associated Kelvin signal. At 20-90-day scale, the highest variance is located over the Indian basin consistent with the MJO events occurrence area. Over Africa and the Guinean gulf the variance pattern looks like the 8-22-day one with a bit lower values, and it coincides with the maxima of the MJO signal over this area. The relative contributions to the 2-90-day variance are weaker than for the 8-22-day band. TRMM variance is also weaker and its maxima still located over the Guinea gulf (not shown).

In the following figures we analyse the time sequences of regression patterns over MarchJune of deseasonalised fields onto the standardised NOAA OLR reference index filtered on 2-8day (Fig.11), 8-22-day (Fig.12) and 20-90-day (Fig.13) respectively. The objective is to analyse for each periodicity band the time sequence of convection, precipitation and associated atmospheric fields, and their links with the Kelvin and MJO equatorial dynamics.

Figure 11 shows the regression patterns from To-3 days to To +3 days of (a) deseasonalised CLAUS Tb, 2.5-8-day CCEK filtered NOAA OLR and deseasonalised divergent $200 \mathrm{hPa}$ wind, (b) deseasonalised TRMM, $925 \mathrm{hPa}$ geopotential height and wind, (c) and 2.5-8-day CCEK filtered NOAA OLR, $925 \mathrm{hPa}$ geopotential height and wind, as well as (d) the associated evolution from To-10 days to To+10 days of the regression coefficients averaged over $2.5^{\circ} \mathrm{S}-7.5^{\circ} \mathrm{N} / 5^{\circ} \mathrm{W}$ $5^{\circ} \mathrm{E}$ for deseasonalised NOAA OLR, filtered 2.5-8-day CCEK NOAA OLR, deseasonalised TRMM, and for the same variables averaged over $2.5^{\circ} \mathrm{S}-7.5^{\circ} \mathrm{N} / 10^{\circ} \mathrm{E}-30^{\circ} \mathrm{E}$ (Central Africa). On Fig.11a,b,c CLAUS Tb has been chosen because it has an evolution similar to NOAA OLR but provides more small scale details. On Fig.11d NOAA OLR has been chosen because the amplitudes can be compared directly to those of filtered CCEK signals. On all panels eastward propagation of convective envelop and associated precipitation is very clear. This dynamics is also signed by a high outflow area of divergent wind at $200 \mathrm{hPa}$ (a). At low levels (b), the patterns of deseasonalised geopotential height and wind are very consistent with a Kelvin-like pattern with a pole of enhanced rainfall located within an area of high easterly geopotential height gradient and of zonal wind convergence, maxima of westerly (resp. easterly) winds being linked to maxima (resp. minima) of geopotential heights. This is confirmed by their similarity with the associated 
Kelvin filtered patterns shown in Fig.11c. The phase speed of the CCEK is estimated to $15 \mathrm{~m} . \mathrm{s}^{-1}$.

546 The mean periodicity of this signal is 5-6 days as seen on Fig.11d both on deseasonalised and

547 Kelvin filtered time series of OLR and precipitation over the Guinean coast. The amplitude of the

548 Kelvin signal is about $40 \%$ of the deseasonalised signal. Over Central Africa the time lag is of one

549 day and the amplitude is highly reduced respect to the Guinean coast index. A last point is that

550 convective activity shown through deseasonalised $\mathrm{Tb}$ is initiated at To-1 also over Nigeria and

551 Cameroon ahead of the CCEK filtered signal and enhances on the spot to cover the whole Guinean

552 coast at To in phase with CCEK.

The results of similar computations from the 8-22-day signal are shown on Fig.12. The evolution and the spatial patterns look like those of 2-8-day signal except wavelength and periodicity are higher, as shown in the distribution of the RCPs on the space-time spectral diagram (Fig.8b). Convective and rainfall envelops are more zonally elongated but still closely associated with a Kelvin-like pattern (Fig.12a,b,c). The phase speed of the CCEK is estimated to $13 \mathrm{~m} . \mathrm{s}^{-1}$. The mean periodicity is 11-12 days in OLR and 10 days in TRMM both on deseasonalised and Kelvin filtered signals. The amplitude of the Kelvin signal is $40 \%$ of the deseasonalised signal. Over Central Africa the time lag is of one day and the amplitude is highly reduced respect to the Guinean coast index. As seen in Fig.4a, a zonal band of negative CCEK NOAA OLR and Tb values is also present along $30^{\circ} \mathrm{N}$ over northern Africa and Saudi Arabia, corresponding to atmospheric moisture or non-precipitating clouds probably associated with tropical plumes. Finally as for the 2.5-8-day signal, convective activity shown through deseasonalised $\mathrm{Tb}$ is initiated at To-2 over the whole Guinean coast a bit ahead of the CCEK filtered signal, enhances on the spot to cover the whole Guinean coast at To in phase with CCEK, and is still present at $\mathrm{To}+2$ while CCEK is centered at $25^{\circ} \mathrm{E}$.

Finally Fig.13 shows results of similar computations but for the 20-90-day and MJO filtered signals. Again in agreement with Fig. $8 \mathrm{~b}$ an eastward propagative signal is present over the whole domain with a higher wavelength associated with a mean periodicity of 28-30 days both on deseasonalised and MJO filtered signals. Over the Indian sector the well-known MJO signal is clearly detected through large $\mathrm{Tb}$ and NOAA OLR anomalies covering the Indian basin and moving eastward. The TRMM signal shows a more northward propagation of an enhanced rainbelt in this Indian sector. The MJO contibution over the Guinean coast is about $60 \%$ of the deseasonalised signal. Again the modulations weaken over Central Africa, especially in terms of precipitation. Finally as seen for the 8-22-day signal, a zonal band of negative CCEK NOAA OLR and $\mathrm{Tb}$ values is also present along $30^{\circ} \mathrm{N}$ over northern Africa and Saudi Arabia, corresponding to atmospheric moisture or non-precipitating clouds probably associated with tropical plumes (Knippertz and Fink 2009).

In conclusion we have shown that the three main modes of convective variability from synoptic to intra-seasonal time scales detected in section 4 are closely associated with eastward propagating convectively coupled Kelvin wave and MJO activity. This confirms that while rainproducing individual systems are moving westward, their activity are highly modulated by subregional and regional scales envelops, all of them moving to the east. This is a burning issue for operational forecasting centers to be able to monitor and predict such eastward propagating envelops of convective activity. It is a way of considering the forecast exercise that is not usual in African forecast centers where the focus is put more on predictability of individual convective systems. However this can provide information about cumulative effects of convective activity or rainfall amount that can occur typically during a sequence of several days to two weeks. Predictability of such sequence may be higher, at least in terms of qualitative or probabilistic forecasts, for instance through the TIGGE ensemble forecast products. 


\section{Westward propagation signals}

Our results have shown the dominance of eastward propagating signals linked to the equatorial atmospheric dynamics at the three main periodicity bands. However Fig.8a shows that westward propagating signals also exist in the Atlantic-Africa domain linked to WIG and CCER waves. Tulich and Kiladis (2012) studied recently the links between WIG and squall lines and showed that convection-wave coupling may be important in this context throughout the tropics including Africa, leading to consider that many squall lines can be classified as convectively coupled inertia-gravity waves with the dispersion properties of shallow-water gravity waves. CCER were also identified in northern summer by Janicot et al. (2010) as one of the main contributors to medium-range to intra-seasonal timescale modes of variability over West Africa. As detailed in the introduction $\mathrm{Yu}$ et al. (2012) extended this investigation on the whole year. They showed that convection associated with CCER wave events is stronger and more frequent during November-April than during May-October in the African monsoon region, but that in general, the MJO events dominate the westward-propagating CCER waves in affecting strong convection in this region.

So we have carried out the same analysis as in section 6 but for these westward signals, in order to evaluate their possible impact on convective activity and their weight relative to eastward propagating signals identified in section 6. Figure 14 shows the same sequences as in Fig. 9 but by superimposing corresponding WIG signals (a) and CCER signals (b and c). Let us notice that for Fig. 14a the WIG filtered CLAUS Tb data have been used instead of NOAA OLR because of its higher space-time resolution more adapted to the higher frequencies of the WIG signal. Paradoxically the consistency between periods of high and low activity of westward propagating convective systems and westward propagating envelops of equatorial waves is less clear than with eastward propagating equatorial signals in this example. Some WIG waves appear clearly associated with full or part of trajectories of convective system but inconsistencies between both are more frequently found. CCER are superimposed on the two other sequences (Fig.14b,c). Again any consistency with the medium-range and intra-seasonal phases of more or less active convection activity is not evident and is less clear than for CCEK and MJO signals.

Figure 15 shows maps of March-June 1979-2010 NOAA OLR variance for filtered 2-8-day (top panel), 8-22-day (middle panel) and 20-90-day (bottom panel) signals, and for superimposed filtered WIG (top panel) and CCER (middle and bottom panels) signals (left). Variance percentages refered to 2-90-day variance are also shown (Fig. 15 right). The patterns of filtered 28-day, 8-22-day and 20-90-day variance have already been described in Fig.10. The WIG variance is by far the highest over West Africa with maxima along $10^{\circ} \mathrm{N}$, that is on the northern margin of the ITCZ, in superimposition to the 2-8-day variance maxima. The CCER variance is the highest over the Indian and West Pacific sectors. Over Africa maxima are located between $10^{\circ} \mathrm{N}$ and $15^{\circ} \mathrm{N}$ (that is far from the ITCZ) and over the equatorial Atlantic including the Guinean gulf in superimposition to 8-22-day and 20-90-day maxima.

Figure 16 panels have been computed as for right panels of Fig.11, 12, 13, but for WIG filtered fields regressed on the standardised 2-8-day filtered NOAA OLR reference index (a) and for CCER filtered fields regressed on standardised 8-22-day filtered NOAA OLR reference index (b) and standardised 20-90-day filtered NOAA OLR reference index (c). The theoretical n=1 WIG has divergence maximised on the equator in phase with antisymmetric meridional flow and in quadrature with symmetric pressure and zonal wind (Kiladis et al. 2009). Tulich and Kiladis (2012) showed that observed composite structures in the Pacific resemble theoretical predictions 
while the African composite displays a more asymmetric structure in the north-south direction with the largest perturbations occurring to the northeast of the composite base point, similar to observations of West African squall lines (see Fig.10 of Tulich and Kiladis 2012). The patterns on Fig.16a are very similar to this description, especially when looking at To, except that the zonal dimension is larger than in Tulich and Kiladis study. The phase speed is around $25 \mathrm{~m} . \mathrm{s}^{-1}$ with an average wavelength of $5500 \mathrm{kms}$ and a mean periodicity of 2.5 days on Fig. 16a which is near the ones observed over the Pacific (29 m.s ${ }^{-1}, 5000 \mathrm{kms}, 2$ days) in Kiladis et al. (2009). However Tulich and Kiladis (2012) observed over north Africa a mean phase speed of $18 \mathrm{~m} . \mathrm{s}^{-1}$. They also performed simulations with WRF model where the control run (with no mean wind) produces a phase speed of $19 \mathrm{~m} . \mathrm{s}^{-1}$ and a run including a typical vertical profile of the north Africa atmosphere a phase speed of $26 \mathrm{~m} \cdot \mathrm{s}^{-1}$. However the amplitude of the WIG is very weak (see the regression time series on Fig.16d). The kinematic differences with the results of Tulich and Kiladis could be due to the different spectral domain filtering. This deserves more investigation but is out of the scope of this paper.

Regression patterns of the CCER filtered fields on the standardised 8-22-day and 20-90day NOAA OLR reference indices are also shown on Fig.16b,c. The fields regressed on the 8-22day index show spatial dynamical structures moving westward close to theoretical CCER waves with cyclonic/anticyclonic cells located north of $10^{\circ} \mathrm{N}$ and rather symmetric structures south of the equator. Along the equator the winds are mainly zonal and converge towards the envelop of enhanced convective activity (see for instance at To-2 and To). The mean wavelength is about $6700 \mathrm{kms}$, the estimated phase speed about $5.4 \mathrm{~m} . \mathrm{s}^{-1}$ and the periodicity around 15 days. The main difference from the theoretical wave is that convection is located along the equatorial band and no signal is evident outside of it. This may be one sign of a weak contribution of CCER to the 8-22day variability.

The fields regressed on the 20-90-day index also show spatial dynamical structures moving westward close to theoretical CCER waves with cyclonic/anticyclonic cells located north of $10^{\circ} \mathrm{N}$ but no symmetric structures south of the equator. The mean wavelength is about $7800 \mathrm{kms}$, the estimated phase speed near $4 \mathrm{~m} . \mathrm{s}^{-1}$ and the periodicity around 24 days. Another difference from the theoretical wave is that convection is located along the equatorial band and no signal is evident outside of it. Again this may be one sign of a weak contribution of CCER to the 8-22-day variability.

We showed in section 4 that the propagation of synoptic to intra-seasonal signals is mainly eastward. In sections 6 and 7 we have decomposed these signals into eastward and westward components. Fig.11d, 12d, 13d enable to evaluate the contributions of CCEK and MJO to the three main modes (2.5-8-day, 8-22-day and 20-90-day), and Fig.16d,e,f the contributions of WIG and CCER to these three main modes. First, looking at the mean periodicities of the signals, the first mode is centred around 5-6 days, consistent with the detected CCEK periodicity but not with the WIG periodicity of 2-2.5 days. The second mode is centred around 11-12 days, consistent with the detected CCEK periodicity but less with the CCER periodicity of 15 days. Finally the third mode is centred around 28-30 days, consistent with the detected MJO periodicity but less with the CCER periodicity of 24 days. The relative contributions on the signals amplitude at To can also be evaluated through these figures. The amplitude at To of the three modes are respectively 14, 14

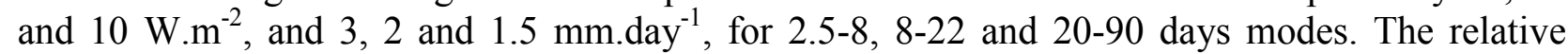
contributions of the equatorial waves at To are, for the $2.5-8$-day signal: $40 \%$ for CCEK and $7 \%$ for WIG, for the 8-22-day signal: $40 \%$ for CCEK and 20\% for CCER, and for the 20-90-day signal: $60 \%$ for $\mathrm{MJO}$ and $15 \%$ for CCER. All these results confirm that eastward propagating 
signals (CCEK and MJO) are highly dominant in the synoptic to intra-seasonal variability of convection and precipitation over the Guinean coast during northern spring.

\section{Conclusion}

This study has proposed an overview of the main synoptic, medium-range and intraseasonal modes of convection and precipitation in northern spring (March-June) over West and Central Africa, and to understand their atmospheric dynamics. Some examples have been presented, showing that mesoscale convective systems can be modulated in terms of occurrences number and intensity at such time scales. Based on EOF analyses on the 2-90-day filtered data, it has been shown that the main mode of convective and rainfall variability is located along the Guinean coast with a moderate to weak extension over Central Africa. Corresponding regressed deseasonalised atmospheric fields highlight an eastward propagation of patterns consistent with convectively coupled equatorial Kelvin wave dynamics. Then a SSA-HAC approach has enabled to define objectively the main spectral bands of variability within the 2-90-day band, separated into three main bands, 2-8-day, 8-22-day and 20-90-day. Within these three bands, space-time spectral decomposition has enabled to identify the relative impacts of CCEK, CCER, MJO and WIG wave dynamics over West and Central Africa. It has been confirmed that eastward propagating signals (CCEK and MJO) are highly dominant in the synoptic to intra-seasonal variability of convection and precipitation over the Guinean coast during northern spring.

We have used different products in terms of longwave irradiance and precipitation. Considering several products instead of only one is interesting because we know that uncertainty is present in these products. So one can get robust conclusions based on common features present within these different data, and have some idea about the related uncertainty of these conclusions.

In conclusion, while rain-producing individual systems are moving westward, their activity are highly modulated by sub-regional and regional scales envelops moving to the east. This is a burning issue for operational forecasting centers to be able to monitor and predict such eastward propagating envelops of convective activity. This study will be also useful as a basis for more detailed further studies, in particular to discriminate the role of local surface-atmosphere interactions from remote forcings in this modes dynamics and to evaluate their predictability. Another issue is the role and impact of these synoptic to intra-seasonal modes of variability on the occurrence of extreme events.

\section{Acknowledgments}

We thank the two anonymous reviewers who helped clarifying some parts of this article. We are also thankful to NOAA-CIRES Climate Diagnostics Center (Boulder, CO) for providing the interpolated OLR dataset from their Web site (online at http://www.cdc.noaa.gov/). The leading author thanks IRD for its $\mathrm{PhD}$ financial support. This study was supported by the French component of AMMA. Based on French initiative, AMMA was built by an international scientific group and is currently funded by a large number of agencies, especially from France, UK, US and Africa. It has been beneficiary of a major financial contribution from the European Community's Sixth Framework Research Programme. Detailed information on scientific coordination and funding is available on the AMMA International website http://www.amma-international.org.

\section{References}

Chauvin, F., R. Roehrig, and J.-P. Lafore, 2010: Intraseasonal variability of the Saharan heat low and its link with midlatitudes. J. Climate, 23, 2544-2561. 
De Coëtlogon, G., S. Janicot and A. Lazar 2010: Intraseasonal variability of the ocean-atmosphere coupling in the gulf of Guinea during northern spring and summer. Quart. J. Roy. Met. Soc. doi:10.1002/qj.554, 136, 426-441. Dee D.P., S.M. Uppala, A.J. Simmons, P. Berrisford, P. Poli, S. Kobayashi, U. Andrae, M.A. Balmaseda, G. Balsamo, P. Bauer, P. Bechtold, A.C.M. Beljaars, L. van de Berg, J. Bidlot, N. Bormann, C. Delsol, R. Dragani, M. Fuentes, A.J. Geer, L. Haimberger, S.B. Healy, H. Hersbach, J.-J. Morcrette, B.-K. Park, C. Peubey, P. de Rosnay, C. Tavolato, J.-N. Thepaut and F. Vitart, 2011: The ERA-Interim reanalysis: configuration and performance of the data assimilation system. Quart. J. Roy. Meteorol. Soc., 137, 553-597.

Ghil, M., and Co-authors, 2002: Advanced spectral methods for climatic series. Rev. Geophys., 40, 1003, doi:10.1029/2000RG000092.

Grueber, A., and A. F. Krueger, 1974: The status of the NOAA outgoing longwave radiation data set. Bull. Amer. Meteor. Soc., 65, 958-962.

Gu G. and R.F. Adler, 2004: Seasonal evolution and variability associated with the West African monsoon. J. Climate, 17, 3364-337.

Gu G., 2008: Intraseasonal variability in the equatorial Atlantic-West Africa during March-June. Climate Dyn., 2008, doi:10.1007/s00382-008-0428-0

Hodges, K. I., D. W. Chappell, G. J. Robinson, and G. Yang, 2000: An improved algorithm for generating global window brightness temperatures from multiple satellite infrared imagery. J. Atmos. Oceanic Technol., 17, 1296-1312.

Huffman, G. J., R. F. Adler, M.M. Morrissey, D. T. Bolvin, S. Curtis, Joyce, B. Mc Gavock, and J. Susskind, 2001: Global precipitation at one-degree daily resolution from multisatellite observations. J. Hydrometeor., 2, 36-50.

Huffman G.J., D.T. Bolvin, E.J. Nelkin, D.B. Wolff, R.F. Adler, G. Gu, Y. Hong, K.P. Bowman and E.F. Stocker, 2007: The TRMM multisatellite precipitation analysis (TMPA): quasi-global, multiyear, combined sensor precipitation estimates at fine scales. J. Hydrometeorol., 8, 38-55.

Janicot, S., C. Thorncroft et al., 2008: Large scale overview of the summer monsoon over the summer monsoon in West Africa during the AMMA field experiment in 2006. Annales Geophysicae, 26, 2569-2595.

Janicot, S., F. Mounier, N.M. Hall, S. Leroux, B. Sultan and G. Kiladis, 2009: The dynamics of the West African monsoon. Part IV: Analysis of 25-90-day variability of convection and the role of the Indian monsoon J. Climate, 22, 1541-1565.

Janicot, S., F. Mounier, S. Gervois, B. Sultan and G. Kiladis, 2010: The dynamics of the West African monsoon. Part V: The role of convectively coupled equatorial Rossby waves. J. Climate, 23, 4005-4024.

Janicot, S., G. Caniaux, F. Chauvin, G. de Coetlogon, B. Fontaine, N. Hall, G. Kiladis, J.-P. Lafore, C. Lavaysse, S. L. Lavender, S. Leroux, R. Marteau, F. Mounier, N. Philippon, R. Roehrig, B. Sultan and C. M. Taylor, 2011: Intra-seasonal variability of the West African monsoon. Atmos. Sci. Lett., Special issue on AMMA, doi:10.1002/as1.280, vol. 12, 1, 58-66.

Kiladis, G. N., C. D. Thorncroft, and N. M. J. Hall, 2006: Three dimensional structure and dynamics of African easterly waves. Part I: Observations. J. Atmos. Sci., 63, 2212-2230.

Kiladis, G.N., M.C. Wheeler, P.T. Haertel and K.H. Straub, 2009: Convectively coupled equatorial waves. Review of Geophysics, 47, RG2003/2009, paper number 2008RG000266.

Knippertz, P. and A.H. Fink, 2009: Prediction of dry-season precipitation in tropical west Africa and its relation to forcing from the extratropics. Weather and Forecasting, 24, 1064-1084, doi:10.1175/2009WAF2222221.1.

Lavender S.L. and Matthews A.J. 2009: Response of the West African monsoon to the MaddenJulian oscillation. J. Climate, 22, 4097-4116, doi:10.1175/2009JCLI2773.1.

Lavender S.L., C.M. Taylor and Matthews A.J. 2010: Coupled Land-atmosphere intraseasonal 
801

802

803

804

805

806

807

808

809

810

811

812

813

814

815

816

817

818

819

820

821

822

823

824

825

826

827

828

829

830

831

832

833

834

835

836

837

838

839

840

variability of the West African monsoon in a GCM. J. Climate, 23, 5557-5571, doi: 10.1175/2010JCLI3419.1

Leduc-Leballeur, M. L. Eymard and G. de Coëtlogon, 2011: Observation of the marine atmospheric boundary layer in the gulf of Guinea during the 2006 boreal spring. Quart. J. Roy. Met. Soc., 137, 992-1003.

Leduc-Leballeur, G. de Coëtlogon and M. L. Eymard, 2013: Air-sea interaction in the gulf of Guinea at intraseasonal timescales: wind bursts and coastal precipitation in boreal spring. Quart. J. Roy. Met. Soc., 139, 387-400.

Liebmann, B., and C. A. Smith, 1996: Description of a complete (interpolated) outgoing longwave radiation dataset. Bull.Amer. Meteor. Soc., 77, 1275-1277.

Maloney E.D. and J. Shaman, 2008: Intraseasonal variability of the West African monsoon and Atlantic ITCZ. J. Climate, 21, 2898-2918, doi:10.1175/2007JCLI1999.1

Matsuno, T., 1966: Quasi-geostrosphic motion in the equatorial area. J. Meteor. Soc. Japan, 44, 25-42.

Matthews, M., 2004: Intraseasonal variability over tropical Africa during northern summer. J. Climate, $17,2427-2440$.

Mekonnen, A., C. D. Thorncroft and A. R. Aiyyer, 2006: Analysis of convection and its association with African easterly waves. J. Climate, 19, 5405-5421.

Mekonnen, A., C. D. Thorncroft, A. R. Aiyyer, and G. N. Kiladis, 2008: Convectively coupled Kelvin waves over tropical Africa during the boreal summer: structure and variability. J. Climate, 21, 6649-6667, doi:10.1175/2008JCLI2008.1.

Mohino, E., S. Janicot, H. Douville and L. Li, 2012: Impact of the Indian part of the summer MJO on West Africa using nudged climate simulations. Climate Dynamics, 38, 2319-2334, doi :10.1007/s00382-011-1206-y

Mounier, F., G. Kiladis and S. Janicot, 2007: Analysis of the dominant mode of convectively coupled Kelvin waves in the West African monsoon. J. Climate, 20, 1487-1503.

Mounier, F., S. Janicot and G. Kiladis, 2008: The West African monsoon dynamics. Part III: The quasi-biweekly zonal dipole. J. Climate, 21, 1911-1928.

Nguyen, H. and J.-P. Duvel, 2008: Synoptic wave perturbations and convective systems over equatorial Africa. J. Climate, 21, 6372-6388, doi: 10.1175/2008JCLI2409.1

Pohl, B., S. Janicot, B. Fontaine and R. Marteau, 2009: Implication of the Madden-Julian Oscillation in the 40-day variability of the West African monsoon, and associated rainfall anomalies. J. Climate, 22, 3769-3785, doi :10.1175/2009JCLI2805.1

Roehrig, R., F. Chauvin and J.-P. Lafore, 2011: 10-25-Day Intraseasonal variability of convection over the Sahel: A role of the Saharan heat low and midlatitudes. J. Climate, 24, 58635878, doi: 10.1175/2011JCLI3960.1

Roundy, P. E., and W. M. Frank, 2004: A climatology of waves in the equatorial region. J. Atmos. Sci., 61, 2105-2132.

Saporta G, 1990: Probabilités, analyses de données et statistique. Ed. Technip, Paris

Straub, K. H., and G. N. Kiladis, 2002: Observations of convectively coupled Kelvin waves in the eastern Pacific ITCZ. J. Atmos. Sci., 59, 30-53.

Sultan, B. et S. Janicot, 2003 : The West African monsoon dynamics. Part II: The pre-onset and the onset of the summer monsoon. J. Climate, 16, 3407-3427.

Taylor CM. 2008: Intraseasonal land-atmosphere coupling in the West African monsoon. J. Climate, 21, 6636-6648.

Tchakoutio Sandjon A., A. Nzeukou and C. Tchawoua, 2012: Intraseasonal atmospheric variability and its interannual modulation in Central Africa. Meteorol. Atmos. Phys., 117, 167179, doi:10.1007/s00703-012-0196-6. 
841 Tulich, S.N. and G.N. Kiladis, 2012: Squall lines and convectively coupled gravity waves in the 842 tropics: Why do most cloud systems propagate westward ? J. Atmos. Sci, 69, 2995-3012, 843 doi:10.1175/JAS-D-11-0297.1

844 Vautard, R., and M. Ghil, 1989: Singular spectrum analysis in nonlinear dynamics with 845 applications to paleoclimatic time series. Physica D, 35, 392-424.

846 Vautard R., P. Yiou, and M. Ghil, 1992: Singular spectral analysis: A toolkit for short, noisy 847 chaotic signals. Physica D, 58, 95-126.

848 Ventrice, M. J., C. D. Thorncroft, and P.E. Roundy, 2011: The Madden Julian Oscillation's 849 influence on African easterly waves and downstream tropical cyclogenesis. Mon. Wea. Rev., 139, $850 \quad 2704-2722$.

851 Ventrice, M. J., C. D. Thorncroft, and M. A. Janiga, 2012a: Atlantic tropical cyclogenesis: A 852 three-way interaction between an African easterly wave, diurnally varying convection, and a 853 convectively-coupled atmospheric Kelvin wave. Mon. Wea. Rev., 140, 1108-1124.

854 Ventrice, M. J., C. D. Thorncroft, and C. J. Schreck III, 2012b: Impacts of convectively coupled 855 Kelvin waves on environmental conditions for Atlantic tropical cyclogenesis. Mon. Wea. Rev., $856140,2198-2214$.

857 Ventrice, M. J., and C. D. Thorncroft, 2013: A convectively coupled equatorial Kelvin wave's 858 impact on African easterly wave activity. Mon. Wea. Rev., in press.

859 Wheeler, M., and G. N. Kiladis, 1999: Convectively coupled equatorial waves: Analysis of clouds 860 and temperature in the wavenumber-frequency domain. J. Atmos. Sci., 56, 374-399.

861 Wheeler, M., G. N. Kiladis and P. J. Webster, 2000: Large scale dynamical fields associated with 862 convectively coupled equatorial waves. J. Atmos. Sci., 57, 613-640.

863 Yang, G. and J. Slingo, 2001: The diurnal cycle in the tropics. Mon. Wea. Rev., 129, 784-801.

864 Yu W., W. Han and D. Gochis, 2012: Influence of the Madden-Julian oscillation and intraseasonal 865 waves on surface wind and convection of the tropical Atlantic ocean. J. Climate, 25, 8057-8074, 866 doi:10.1175/JCLI-D-11-00528.1 
Table 1: First row: Correlations between the reference 2-90-day filtered NOAA OLR index over the Guinean coast and similar indices computed from the five other OLR and rainfall variables. Second row: Correlations of the RCPs reconstructed NOAA OLR index over the 20-90-day band and of the other 20-90-day band filtered indices with the 20-90-day NOAA OLR reference index. Third row: As second row but for the 8-22-day band. Fourth row: As second row but for the 2-8day band.

877

Table 2: Percentages of explained variance of the first 30 T-EOFs of the SSA.

880

881

882

883

884

885

886

887

888

889

890

891

892

893

894

895

896

897

898

899

900

901

902

903

904

905

906

907

908

909

910

911

912

913

914

915

916

917

\section{Figures captions}

Figure 1: (a) Mean bi-monthly TRMM rainfall (mm.day ${ }^{-1}$, color) and NOAA OLR (W.m ${ }^{-2}$, contour) from January-February to November-December. (b) Time-latitude cross-sections of TRMM (color) and NOAA OLR (contour) averaged over $10^{\circ} \mathrm{W}-10^{\circ} \mathrm{E}$ (left) and $10^{\circ} \mathrm{E}-30^{\circ} \mathrm{E}$ (right).

Figure 2: Examples of characteristic (a) synoptic, (b) medium-range and (c) intra-seasonal time sequences of convection and precipitation over the Atlantic-Africa domain. The time-longitude cross-sections show the CLAUS Tb values $\left({ }^{\circ} \mathrm{K}\right)$ along $45^{\circ} \mathrm{W}-45^{\circ} \mathrm{E}$ and averaged over $2.5^{\circ} \mathrm{S}-$ $7.5^{\circ} \mathrm{N}$; it highlights westward propagating convective systems. Curves represent the TRMM rainfall values $(\mathrm{mm})$ averaged over $2.5^{\circ} \mathrm{S}-7.5^{\circ} \mathrm{N} / 5^{\circ} \mathrm{W}-5^{\circ} \mathrm{E}$. The $5^{\circ} \mathrm{W}-5^{\circ} \mathrm{E}$ domain is displayed on the cross-sections. Corresponding wavelet diagrams of a NOAA OLR index computed as an average over $2.5^{\circ} \mathrm{S}-7.5^{\circ} \mathrm{N} / 5^{\circ} \mathrm{W}-5^{\circ} \mathrm{E}$ are shown below $(\mathrm{d}, \mathrm{e}, \mathrm{f})$. Three time sequences are presented : (a) the sequence from 1 to 30 March 1999 where the synoptic timescale signal is well defined and daily rainfall evolution is smoothed by a moving-sum over 2 days; (b) the sequence from 25 March to 5 May 2001 where the medium-range timescale signal is well defined and daily rainfall evolution is smoothed by a moving-sum over 5 days; (c) the sequence from 1 March to 1 July 2003 where the intra-seasonal timescale signal is well defined and daily rainfall evolution is smoothed by a moving-sum over 10 days.

Figure 3: Variance fields of the reconstruction by the first principal component of March-June 290-day filtered (a) NOAA OLR $\left(\mathrm{W}^{2} \cdot \mathrm{m}^{-4}\right)$, (b) CLAUS Tb $\left({ }^{\circ} \mathrm{K}^{2}\right)$, (c) ERAI OLR $\left(\mathrm{W}^{2} \cdot \mathrm{m}^{-4}\right)$, and (d) GPCP, (e) TRMM, (f) ERAI rain $\left(\mathrm{mm}^{2} \cdot\right.$ day $\left.^{-2}\right)$. Respective percentages of explained variance by the first component are indicated. Color scales are different so as to better see the details.

Figure 4a: Regression patterns over March-June onto the standardised 2-90-day filtered NOAA OLR reference index (average over $2.5^{\circ} \mathrm{S}-7.5^{\circ} \mathrm{N} / 5^{\circ} \mathrm{W}-5^{\circ} \mathrm{E}$ ) of deseasonalised variables for their respective periods (see section 2). (a) at time To (with no time lag) for (a) NOAA OLR, (b) CLAUS Tb, (c) ERA OLR (W.m ${ }^{-2}$ ) with superimposed $925 \mathrm{hPa}$ wind and geopotential height, and for (d) GPCP, (e) TRMM, (f) ERAI rain $\left(\mathrm{mm}^{\left.- \text {day }^{-1}\right)}\right.$ with superimposed $200 \mathrm{hPa}$ divergent wind and velocity potential. Only $90 \%$ significant OLR and rainfall regression coefficients are displayed, and for a better clarity all wind, geopotential height and velocity potential regression coefficients are displayed. Vector scales are displayed.

Figure 4b: Same as Fig.4a but for To-20 days/To+20 days time-longitude cross-sections averaged over $2.5^{\circ} \mathrm{S}-7.5^{\circ} \mathrm{N}$ (regression coefficients of zonal wind components are displayed). 
918 Figure 5: March-June spectra of the 2-90-filtered (a) NOAA OLR reference index and of other 919 filtered indices computed over the same area $2.5^{\circ} \mathrm{S}-7.5^{\circ} \mathrm{N} / 5^{\circ} \mathrm{W}-5^{\circ} \mathrm{E}$ ( (b) CLAUS Tb, (c) ERAI 920 OLR, (d) GPCP, (e) TRMM and (f) ERAI rain) on their respective periods (see section 2). Green, 921 blue and red curves represent the red noise spectrum and the associated significance level of $90 \%$

Figure 6: Spectra of the 2-90-day filtered NOAA OLR reference index and of the reconstructed signals by pairs of components $\mathrm{RC}_{1-2}$ to $\mathrm{RC}_{29-30}$. The explained variance percentages of the reference index are indicated for every pairs.

Figure 7: Dendrogram of the $\mathrm{RC}_{1-2}$ to $\mathrm{RC}_{29-30}$ spatial regression patterns. The ordinate represents the aggregation index scale based on the intra-classes variance with the Ward distance metric. The evolution of this aggregation index is shown by the dotted line.

Figure 8a: Regions of wavenumber-frequency filtering calculated for February-July over 19792010 for (a) NOAA OLR and respective available periods for the other OLR and rain products ((b) to (f)). Contours show the symmetric power divided by a background spectrum [note that the background was calculated for the full period; see Wheeler and Kiladis (1999) for details on the computation techniques]. Contour interval of this ratio is 0.1 starting at 1.0 , with shading above 1.1 indicative of statistically significant signals. The thin lines are the various equatorial wave dispersion curves for the eight different equivalent depths 2, $58,12,25,50,90$ and $180 \mathrm{~m}$ for Kelvin and Equatorial Rossby waves, and 2, 5 8, 12, $25 \mathrm{~m}$ for Inertio-Gravity waves.

Figure 8b: The boxes indicate the regions of the wavenumber-frequency domain used for filtering of the data to retrieve the longitude-time information of the different convectively coupled equatorial waves [CCEK in blue, CCER in green, WIG in dotted green, and MJO in dotted blue]. Red dots represent the 15 RCs modes computed in section 4 with size proportional to their variance.

Figure 9: Same as Fig.2 including superimposed filtered signals: (a) 2-8-day filtered NOAA OLR signal (red contours) and 2.5-8-day CCEK filtered NOAA OLR signal (black contours); (b) 8-22day filtered NOAA OLR signal (red contours) and 8-22-day CCEK filtered NOAA OLR signal (black contours); (c) 20-90-day filtered NOAA OLR signal (red contours) and MJO filtered NOAA OLR signal (black contours).

Figure 10: Left panels: Maps of March-June 1979-2010 NOAA OLR variance for filtered 2-8day, 8-22-day and 20-90-day signals (colour), and for superimposed filtered 2.5-8-day CCEK, 822-day CCEK and MJO signals respectively. Right panels: Same as left but for variance percentage refered to 2-90-day variance.

Figure 11: Regression patterns over March-June onto the standardised 2-8-day filtered NOAA OLR reference index of variables over their respective periods from To-3 days to To+3 days by 1day step. (a) Deseasonalised CLAUS Tb (colour), 2.5-8-day CCEK filtered NOAA OLR (contours) and deseasonalised divergent $200 \mathrm{hPa}$ wind. (b) Deseasonalised TRMM (colour), 925 hPa geopotential height (contours) and wind. (c) 2.5-8-day CCEK filtered NOAA OLR (colour), $925 \mathrm{hPa}$ geopotential height (contours) and wind. Only $90 \%$ significant NOAA OLR, CLAUS Tb and TRMM regression coefficients are displayed. For a better clarity all wind and geopotential height regression coefficients are displayed. Vector scales are displayed. (d) Evolution from To-10 days to To+10 days of the regression coefficients averaged over $2.5^{\circ} \mathrm{S}-7.5^{\circ} \mathrm{N} / 5^{\circ} \mathrm{W}-5^{\circ} \mathrm{E}$ for deseasonalised NOAA OLR (red "GG"), filtered 2.5-8-day CCEK NOAA OLR (red 
"GG/Kelvin"), deseasonalised TRMM (blue "GG"), and for the same variables (red "CA", red "CA/Kelvin" and blue "CA" respectively) averaged over $2.5^{\circ} \mathrm{S}-7.5^{\circ} \mathrm{N} / 10^{\circ} \mathrm{E}-30^{\circ} \mathrm{E}$.

Figure 12: Same as Fig.11 but for 8-22-day (and 8-22-day CCEK) filtered signals instead of 2-8day (and 2.5-8-day CCEK) signals from To-6 days to To+6 days by 2-day step.

973

974

975

976

977

978

979

980

981

982

983

984

985

986

987

988

989

990

991

992

993

994

995

996

997

998

999

1000

1001

1002

Figure 13: Same as Fig.11 but for 20-90-day (and MJO) filtered signals instead of 2-8-day (and 2.5-8-day CCEK) signals from To-12 days to To +12 days by 4-day step, and from To-20 days to To+20 days for Fig. $13 b$.

Figure 14: Same as Fig.9 but for superimposed filtered WIG signal (a) and CCER signals ((b) and (c)). For (a) the WIG filtered CLAUS Tb data have been used instead of NOAA OLR because of the higher space-time resolution.

Figure 15: Same as Fig.10 but for superimposed filtered WIG (top panel) and CCER (middle and bottom panels) signals.

Figure 16: (a) Regression patterns from To-3 days to To+3 days by 1-day step over March-June onto the standardised 2-8-day filtered NOAA OLR reference index of WIG filtered NOAA OLR (colour), $925 \mathrm{hPa}$ geopotential height (contours) and wind. (b) Regression patterns from To-6 days to To+6 days by 2-day step over March-June onto the standardised 8-22-day filtered NOAA OLR reference index of CCER filtered NOAA OLR (colour), $925 \mathrm{hPa}$ geopotential height (contours) and wind. (c) Regression patterns from To-12 days to To+12 days by 4-day step over March-June onto the standardised 20-90-day filtered NOAA OLR reference index of CCER filtered NOAA OLR (colour), $925 \mathrm{hPa}$ geopotential height (contours) and wind. Only 90\% significant NOAA OLR regression coefficients are displayed. For a better clarity all wind and geopotential height regression coefficients are displayed. Vector scales are displayed. (d) Evolution from To-10 days to To +10 days of the regression coefficients of Fig. 16 a left averaged over $2.5^{\circ} \mathrm{S}-7.5^{\circ} \mathrm{N} / 5^{\circ} \mathrm{W}-5^{\circ} \mathrm{E}$ for deseasonalised NOAA OLR (red "GG"), filtered WIG NOAA OLR (red "GG/WIG"), deseasonalised TRMM (blue "GG"), and for the same variables (red "CA", red "CA/WIG" and blue "CA" respectively) averaged over $2.5^{\circ} \mathrm{S}-7.5^{\circ} \mathrm{N} / 10^{\circ} \mathrm{E}-30^{\circ} \mathrm{E}$. (e) Same as (d) but for Fig. $16 \mathrm{~b}$ middle regression coefficients and CCER instead of WIG. (f) Same as Middle but for Fig.16c right regression coefficients and from To-20 days to To +20 days. 


\begin{tabular}{|c|c|c|c|c|c|c|}
\hline Index & $\begin{array}{c}\text { NOAA } \\
\text { OLR }\end{array}$ & $\begin{array}{c}\text { CLAUS } \\
\text { Tb }\end{array}$ & $\begin{array}{c}\text { ERAI } \\
\text { OLR }\end{array}$ & $\begin{array}{c}\text { GPCP } \\
\text { Rain }\end{array}$ & $\begin{array}{c}\text { TRMM } \\
\text { Rain }\end{array}$ & $\begin{array}{c}\text { ERAI } \\
\text { Rain }\end{array}$ \\
\hline Correl NOAA OLR / other indices & +1.00 & +0.95 & +0.71 & -0.79 & -0.51 & -0.43 \\
\hline $\begin{array}{c}\text { Correl 20-90-day RCPs NOAA } \\
\text { OLR / RCPs other indices }\end{array}$ & +0.98 & +0.94 & +0.85 & -0.88 & -0.84 & -0.69 \\
\hline $\begin{array}{c}\text { Correl 8-22-day RCPs NOAA } \\
\text { OLR / RCPs other indices }\end{array}$ & +0.98 & +0.94 & +0.79 & -0.84 & -0.84 & -0.62 \\
\hline $\begin{array}{c}\text { Correl 2-8-day RCPs NOAA OLR } \\
\text { / RCPs other indices }\end{array}$ & +0.80 & +0.76 & +0.53 & -0.56 & -0.52 & -0.31 \\
\hline
\end{tabular}

1005

1006

\begin{tabular}{|c|c|c|}
\hline Eigenvalue & Variance (\%) & $\begin{array}{c}\text { Cumulated } \\
\text { variance }(\%)\end{array}$ \\
\hline 1 & 6.22 & 6.22 \\
\hline 2 & 5.91 & 12.13 \\
\hline 3 & 4.63 & 16.76 \\
\hline 4 & 4.38 & 21.13 \\
\hline 5 & 4.00 & 25.13 \\
\hline 6 & 3.92 & 29.06 \\
\hline 7 & 3.58 & 32.64 \\
\hline 8 & 3.52 & 36.16 \\
\hline 9 & 3.13 & 39.29 \\
\hline 10 & 3.10 & 42.39 \\
\hline 11 & 2.97 & 45.36 \\
\hline 12 & 2.92 & 48.27 \\
\hline 13 & 2.80 & 51.07 \\
\hline 14 & 2.77 & 53.84 \\
\hline 15 & 2.68 & 56.53 \\
\hline 16 & 2.68 & 59.21 \\
\hline 17 & 2.49 & 61.69 \\
\hline 18 & 2.46 & 64.15 \\
\hline 19 & 2.41 & 66.56 \\
\hline 20 & 2.27 & 68.84 \\
\hline 21 & 2.24 & 71.08 \\
\hline 22 & 2.15 & 73.23 \\
\hline 23 & 1.79 & 75.01 \\
\hline 24 & 1.58 & 76.60 \\
\hline 25 & 1.48 & 78.06 \\
\hline 26 & 1.32 & 79.38 \\
\hline 27 & 1.18 & 80.55 \\
\hline 28 & 1.04 & 81.59 \\
\hline 29 & 1.03 & 82.62 \\
\hline 30 & 1.01 & 83.63 \\
\hline
\end{tabular}

Table 2

Table 1 
1010
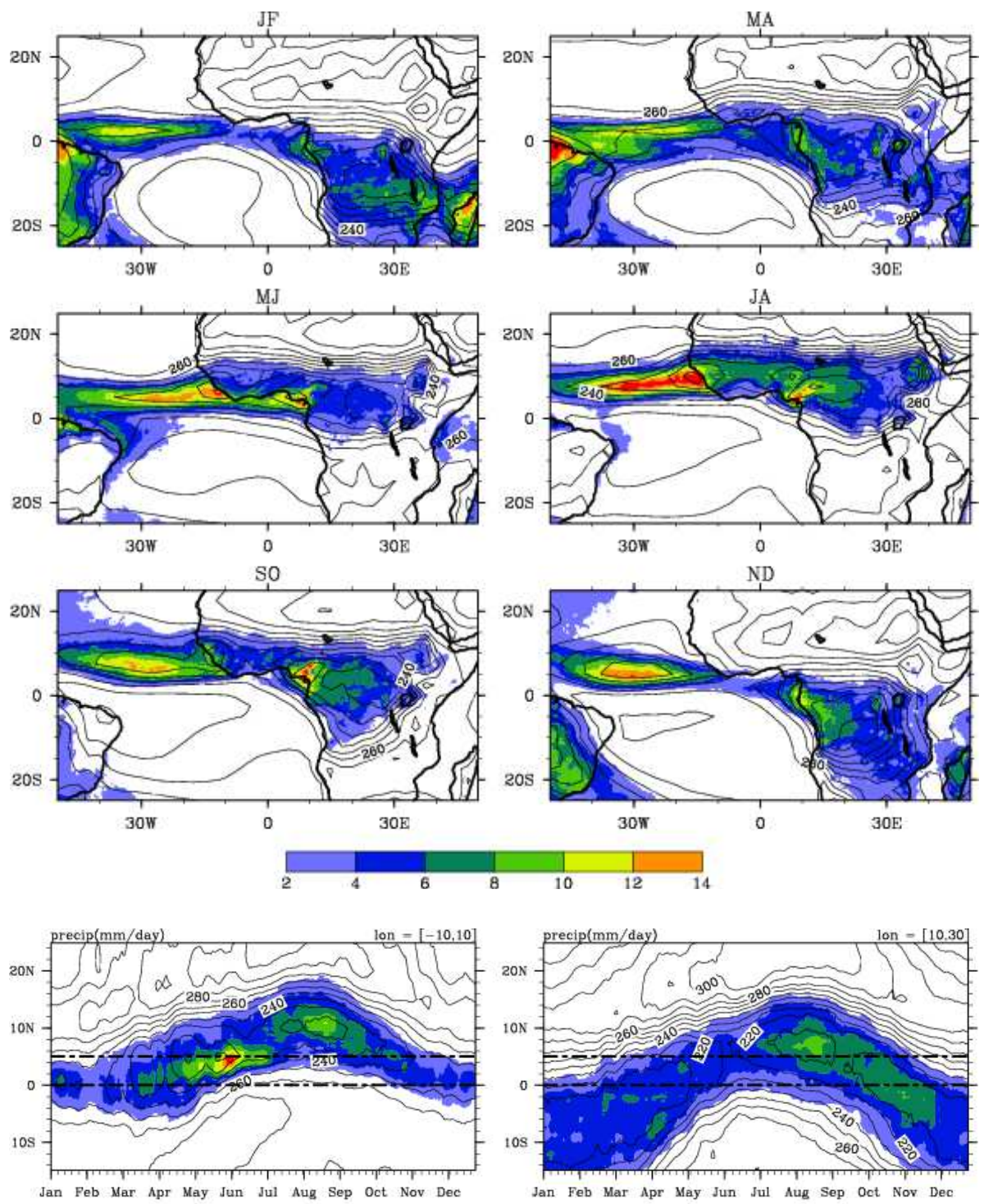

1011

Figure 1

1012 
1013
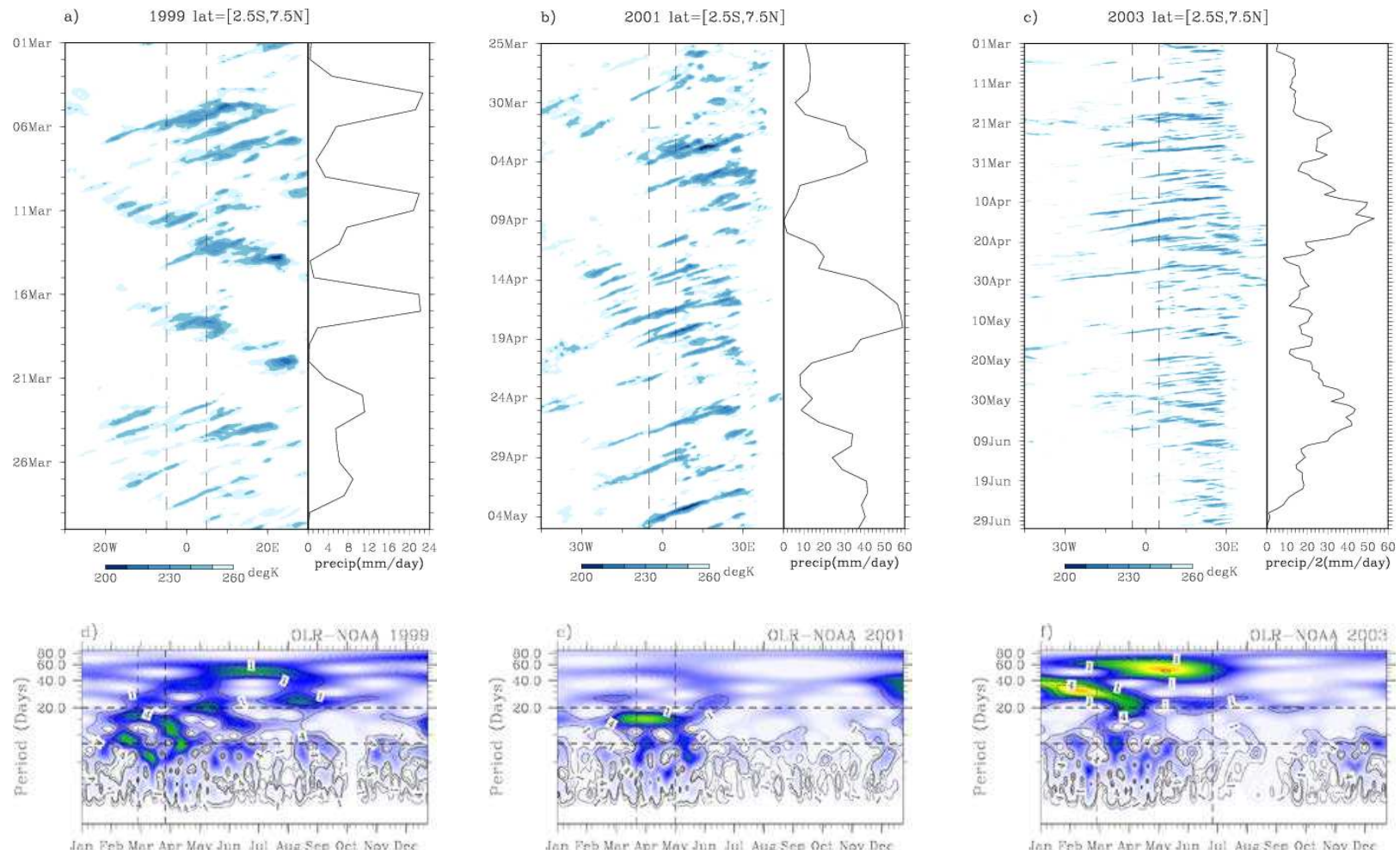

Figure 2

1014

1015

1016
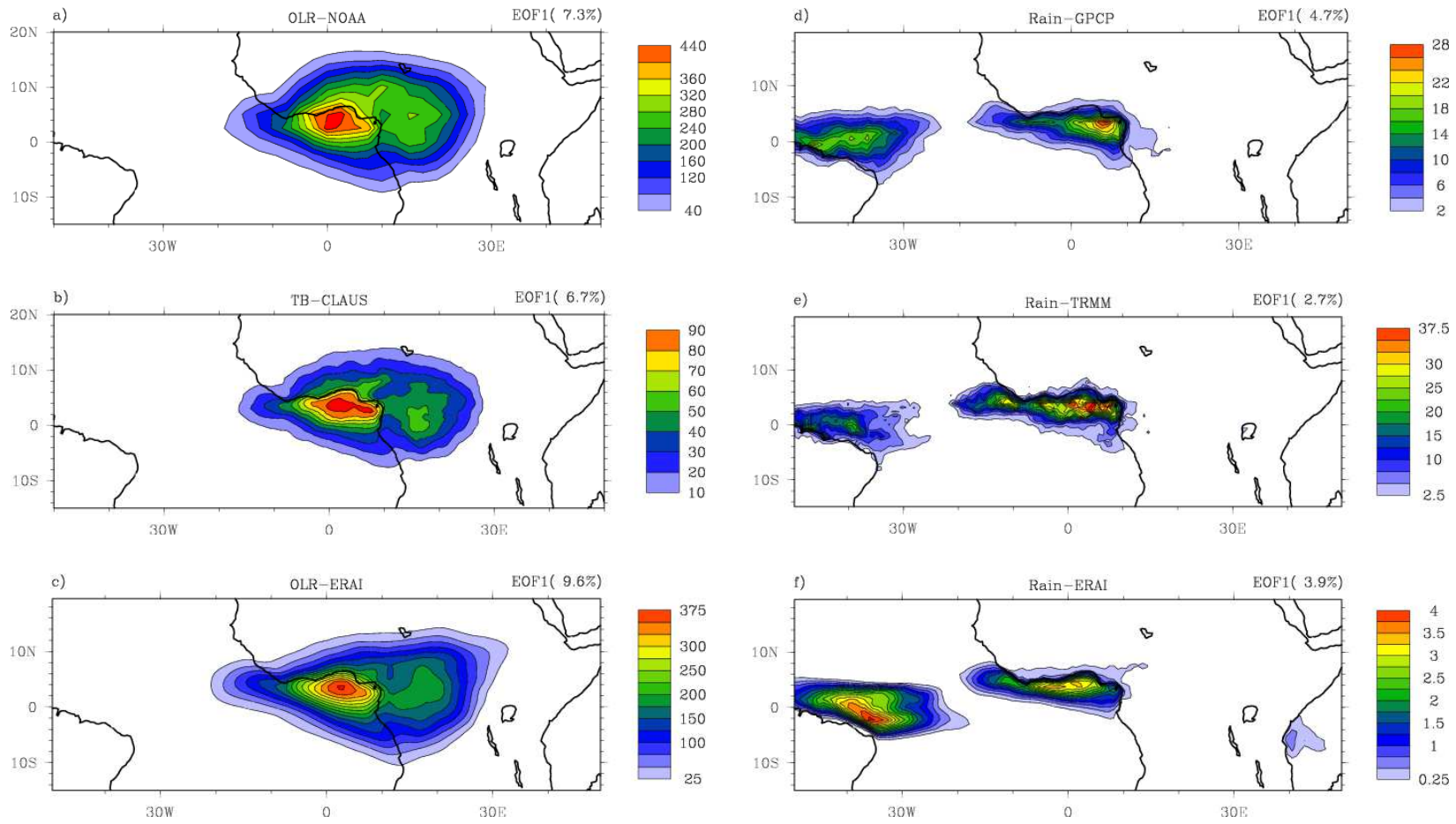

Figure 3

1018 

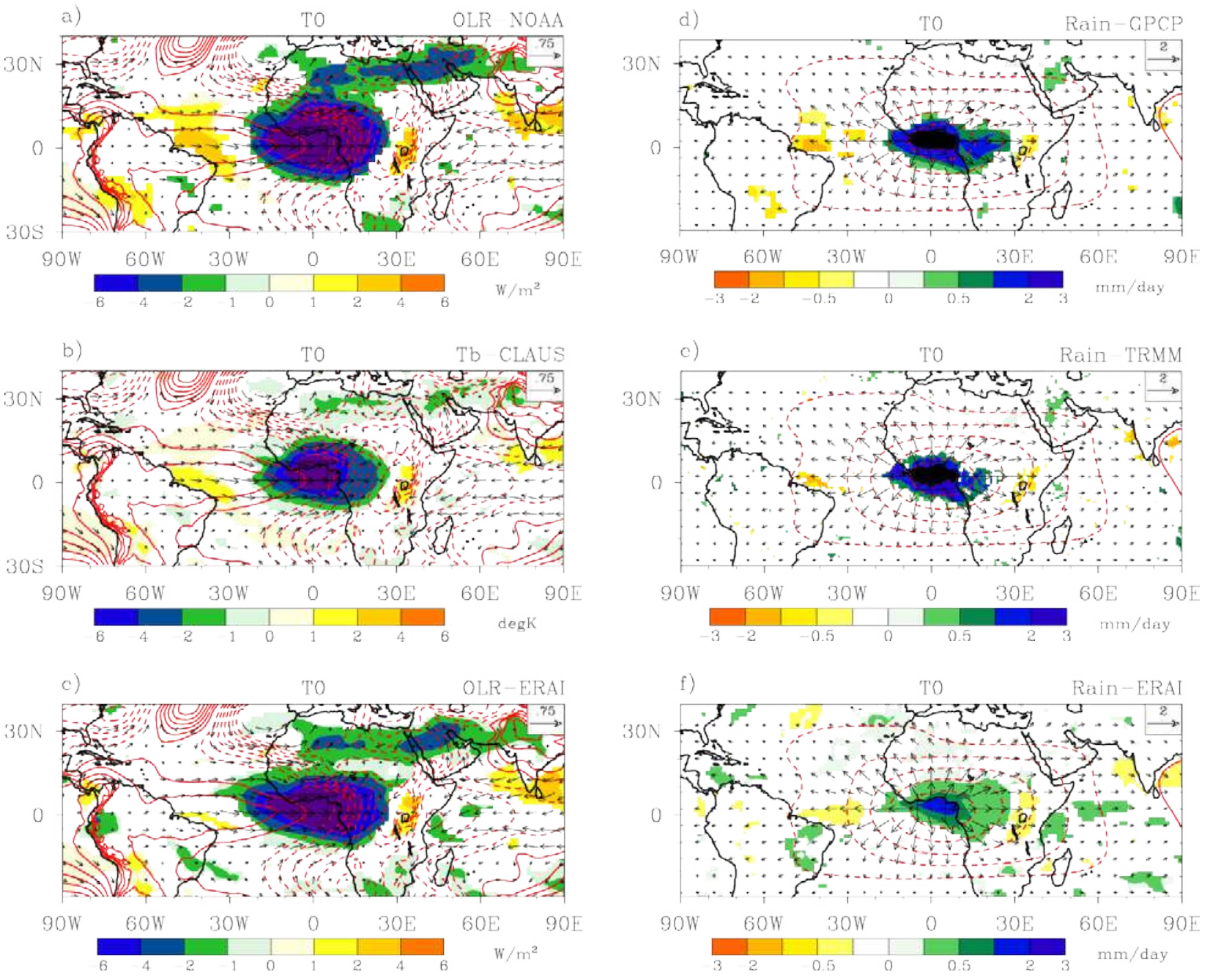

Figure 4a

1019
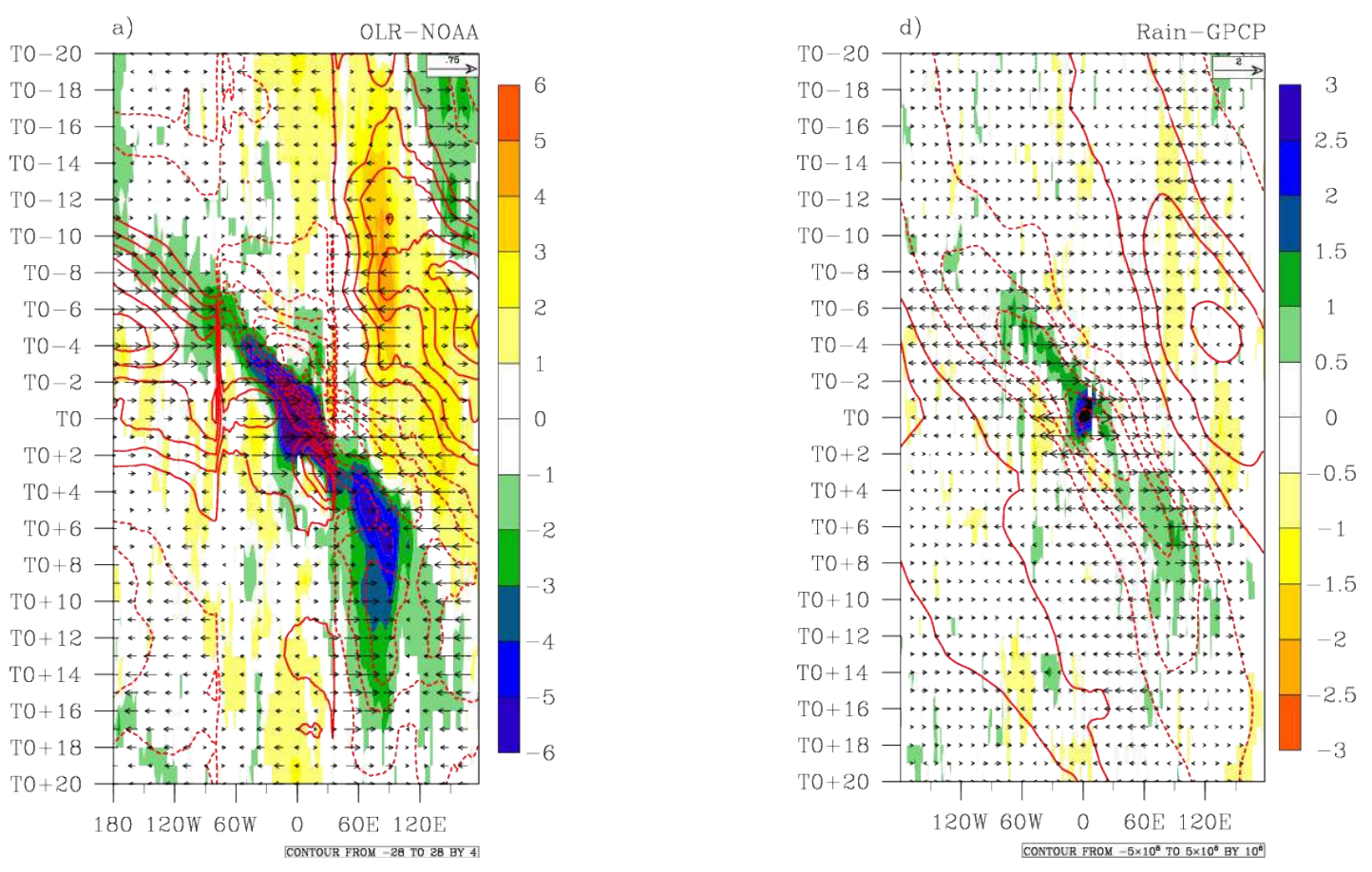

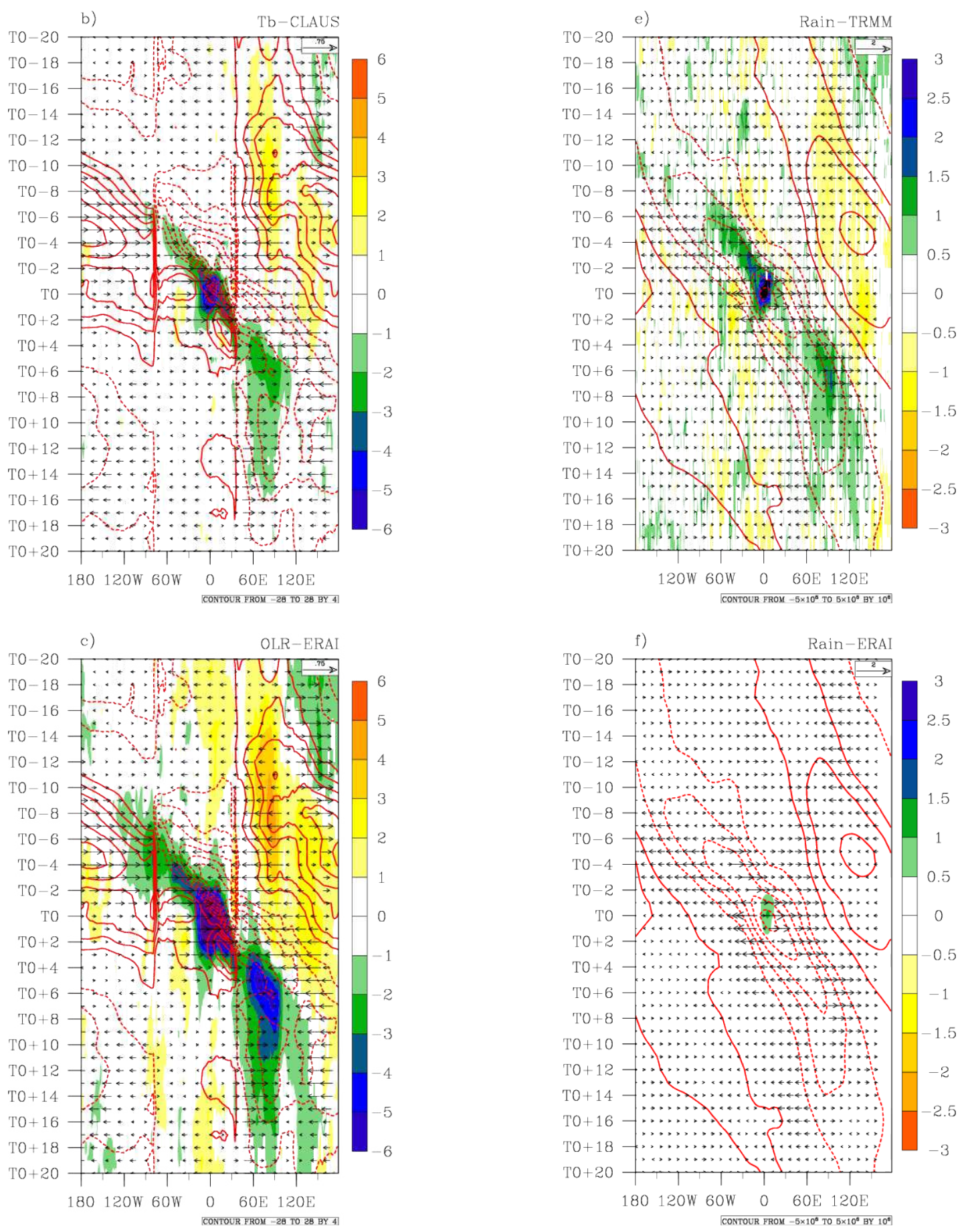

\section{Figure 4b}


1022
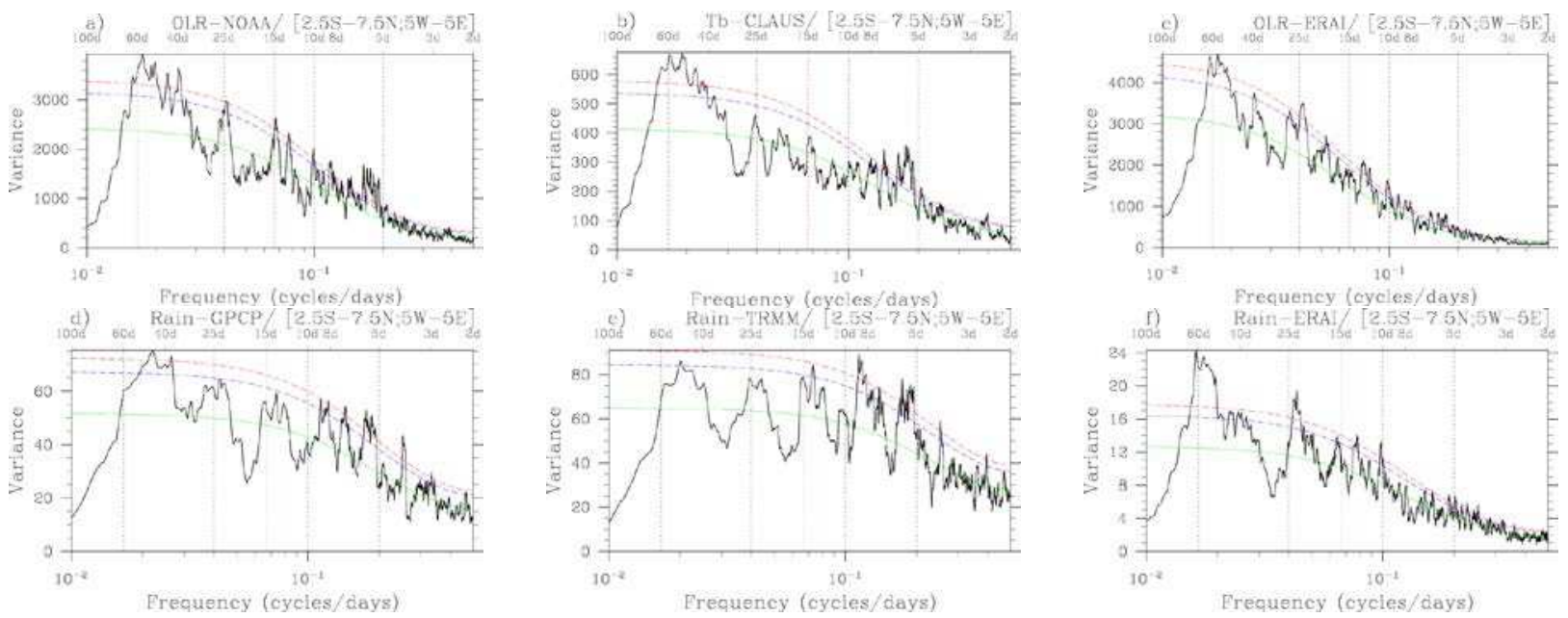

Figure 5

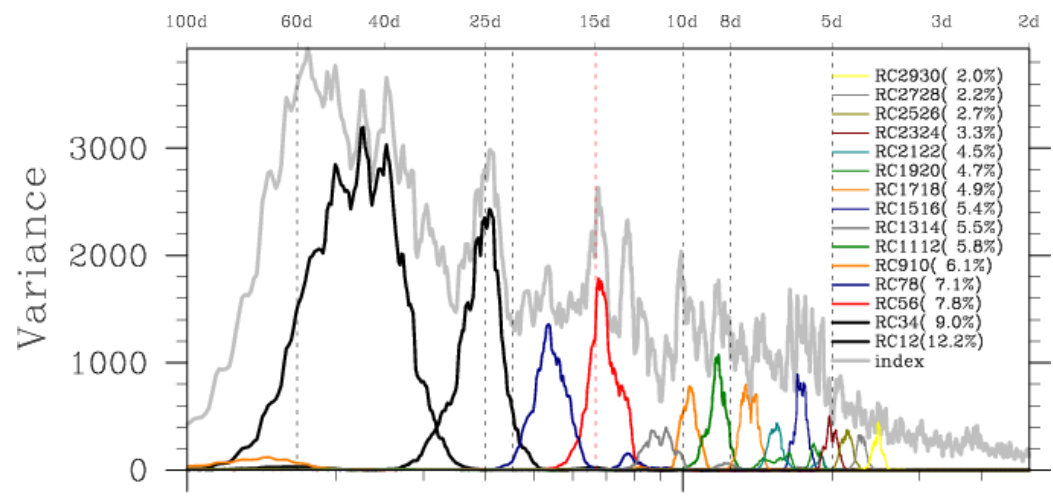

$10^{-2}$

$10^{-1}$

Frequency (cycles/days)

1023

Figure 6

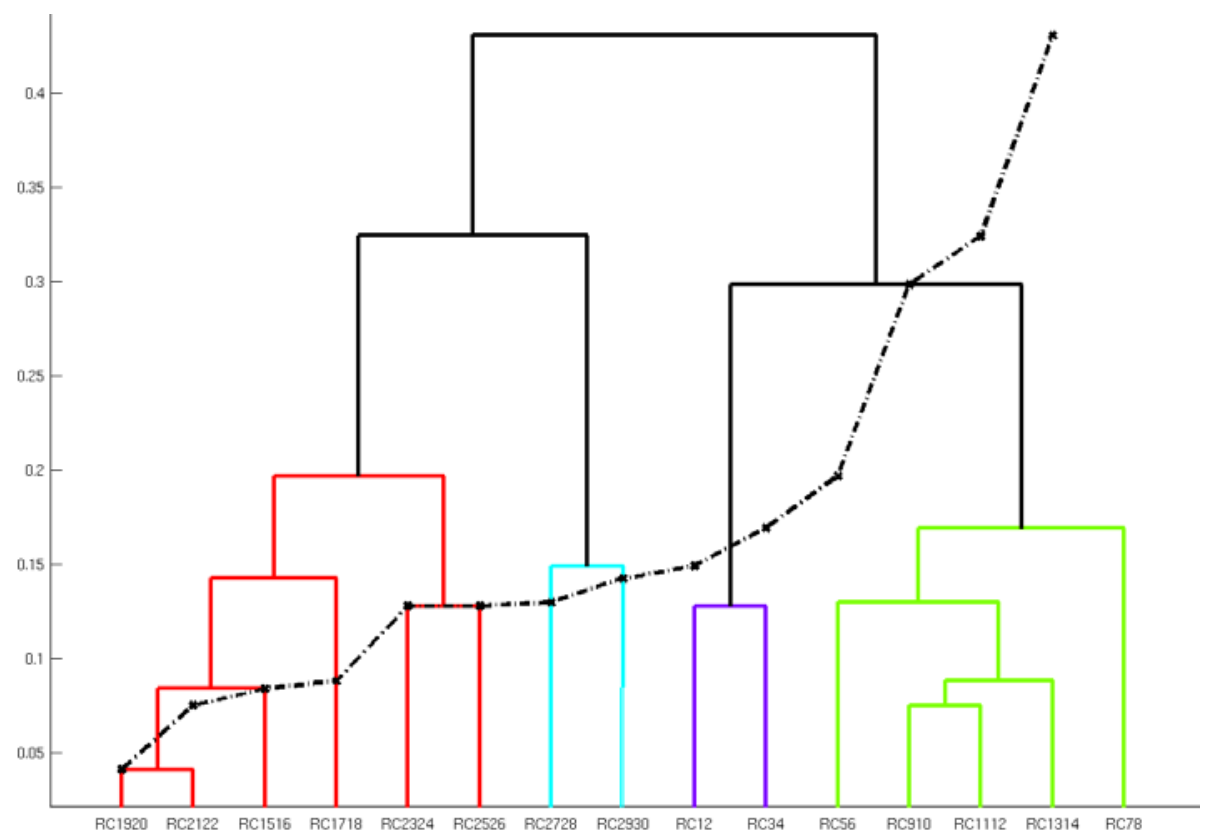

Figure 7 


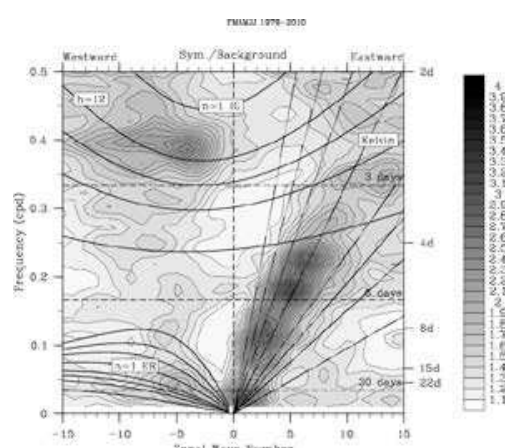

(a) NOAA OLR

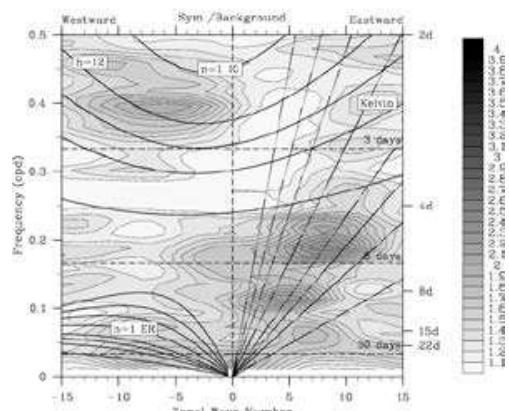

(d) GPCP Rain

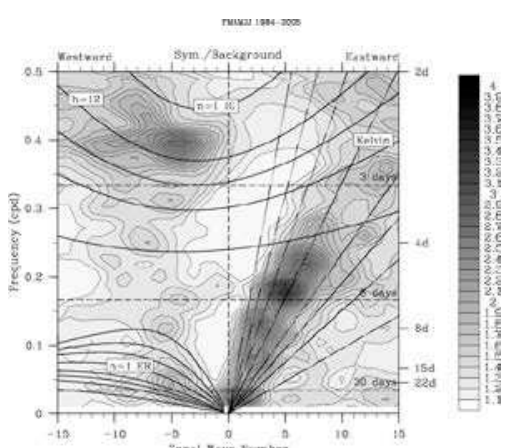

(b) CLAUS Tb

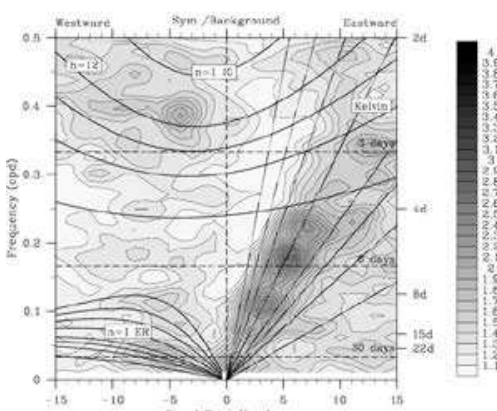

(e) TRMM Rain

Figure 8a

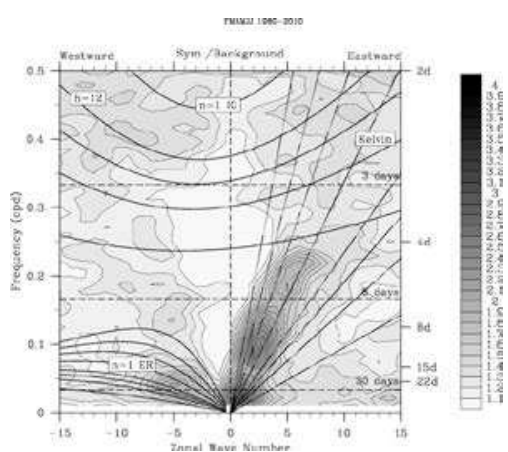

(c) ERAI OLR

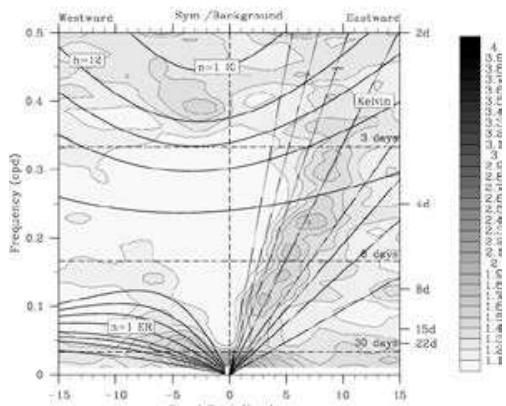

(f) ERAI Rain

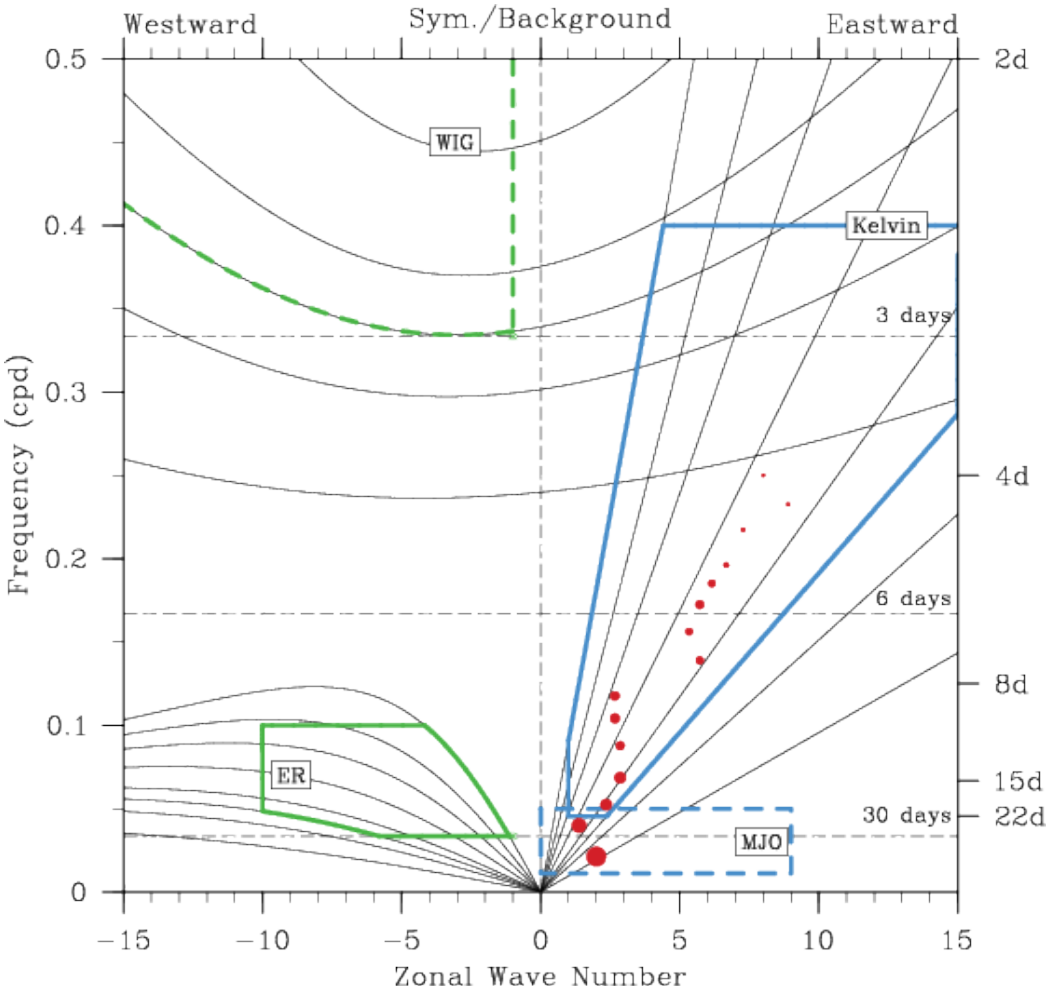

Figure 8b 
1031

a) 1999 lat $=[2.5 \mathrm{~S}, 7.5 \mathrm{~N}]$

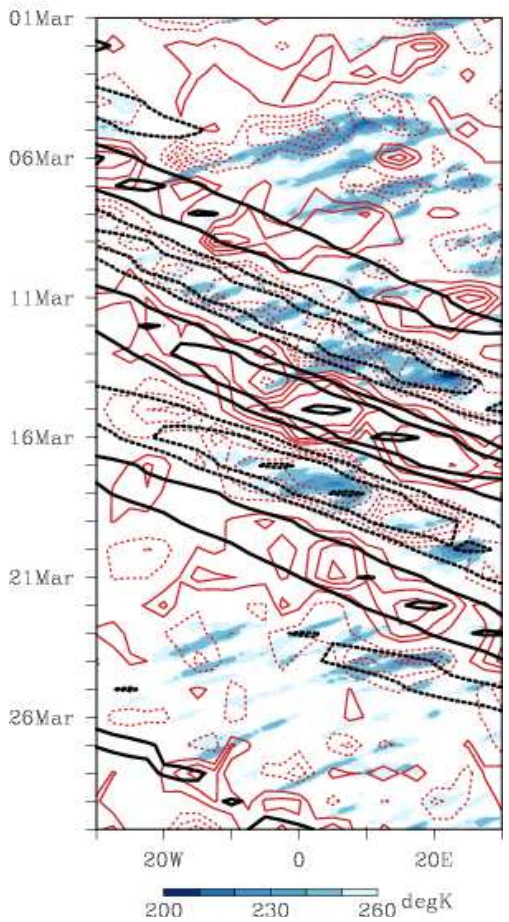

b)

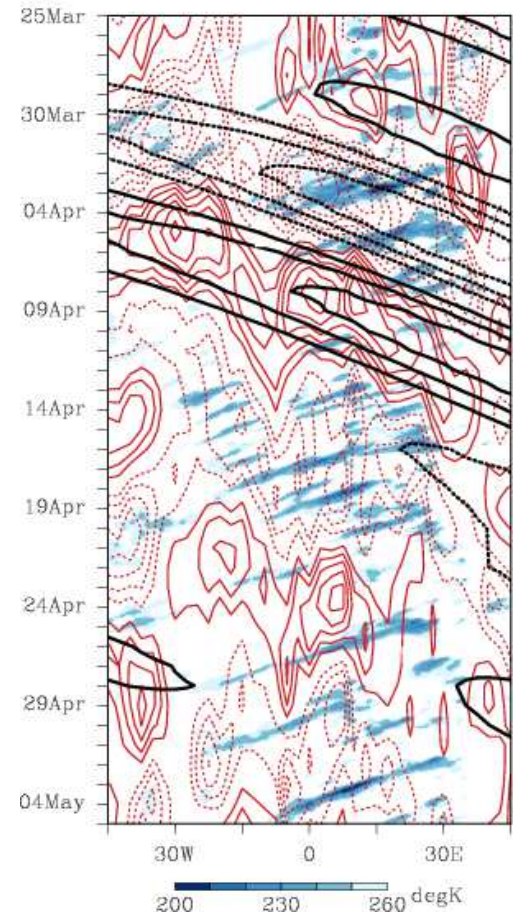

Figure 9 c)

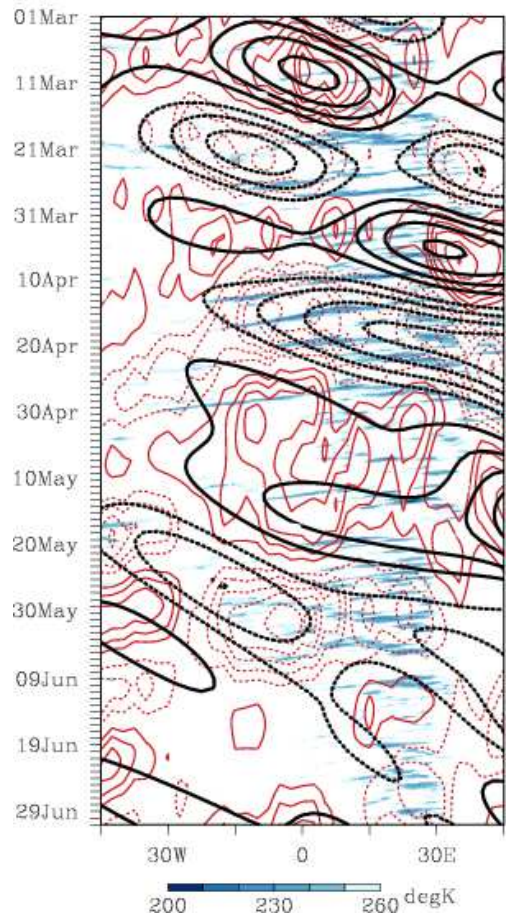

1032
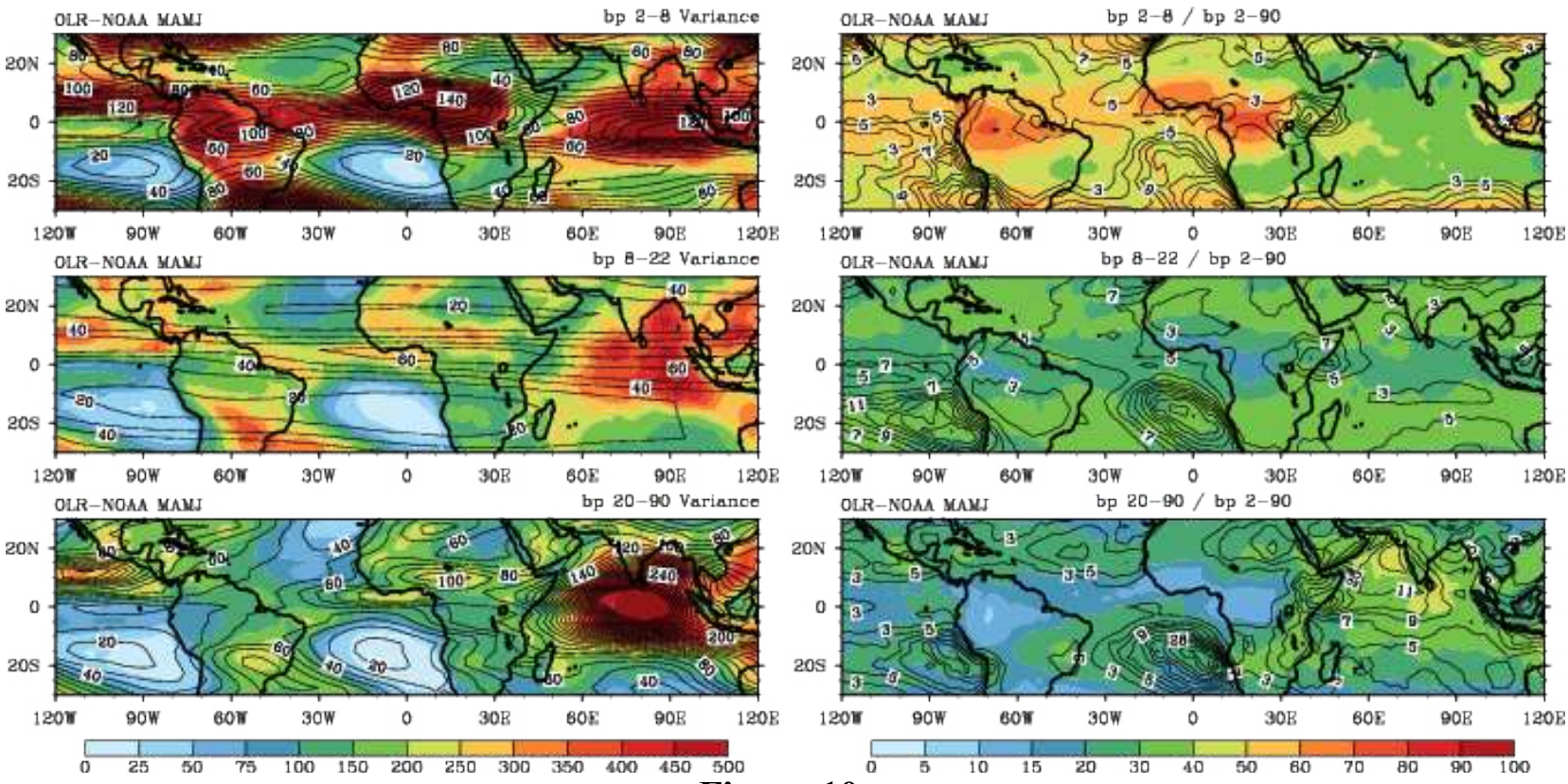

Figure 10

1034 
1035
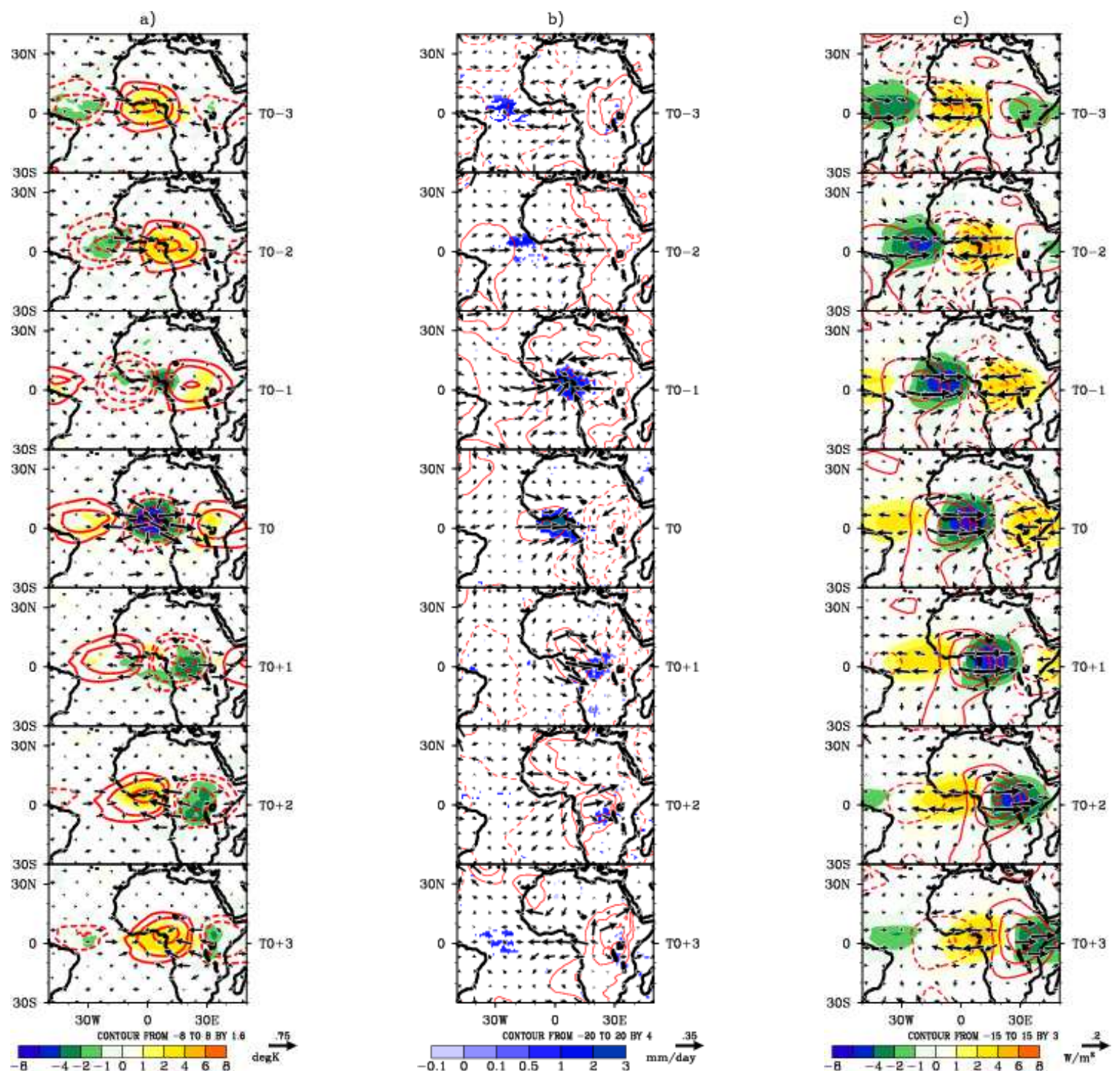

1036

1037

1038

1039

d)

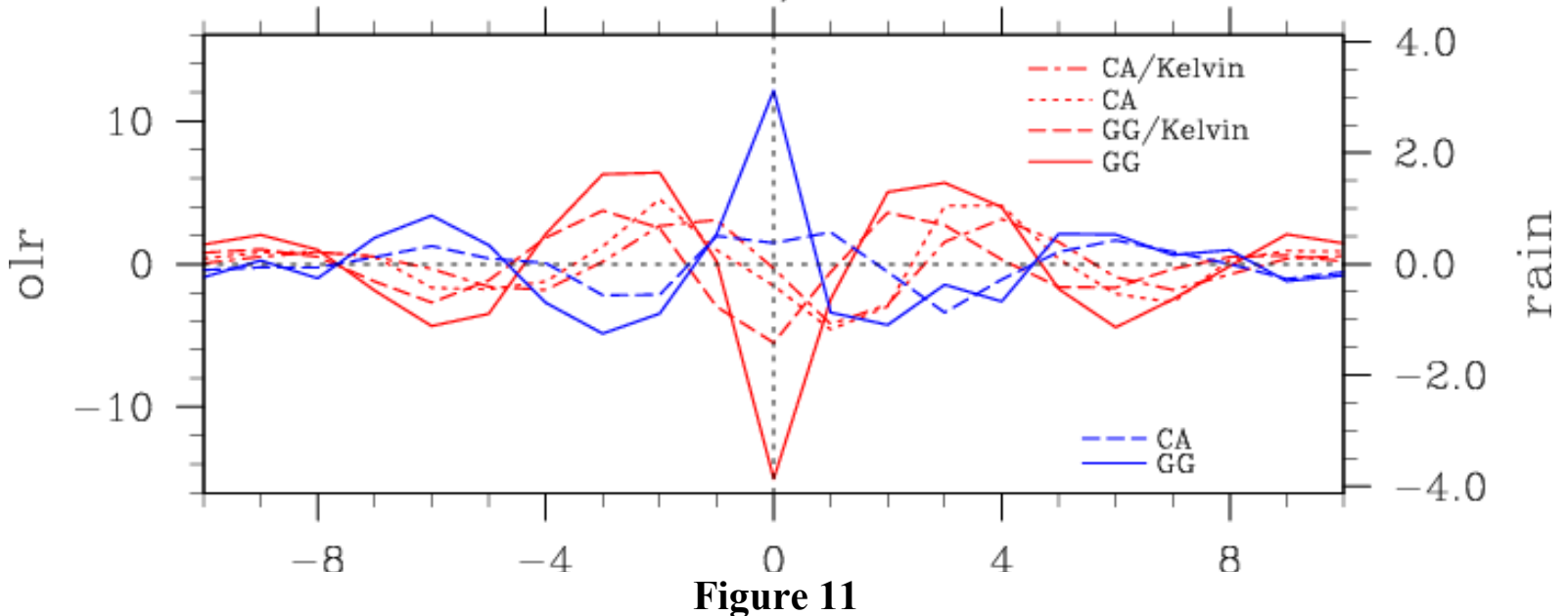

Figure 11 
1040
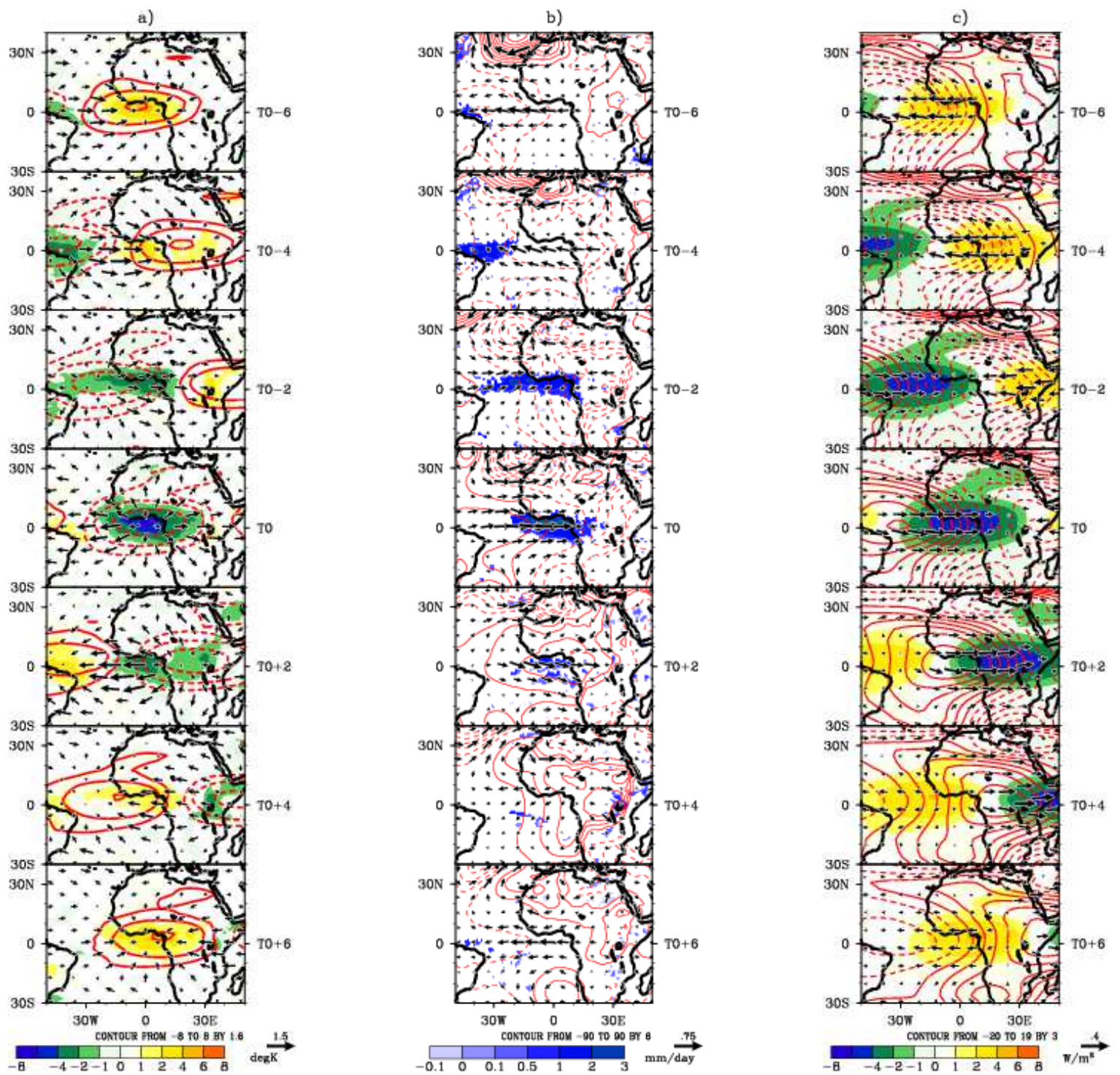

d)

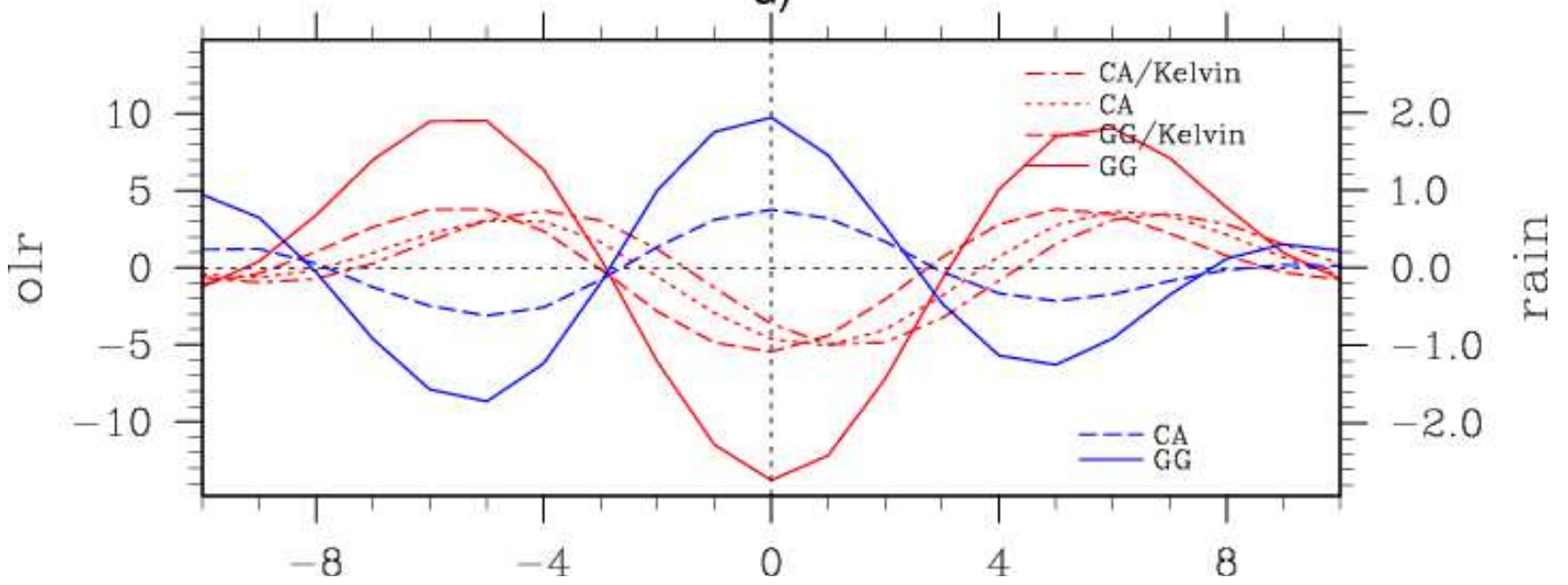

Figure 12 

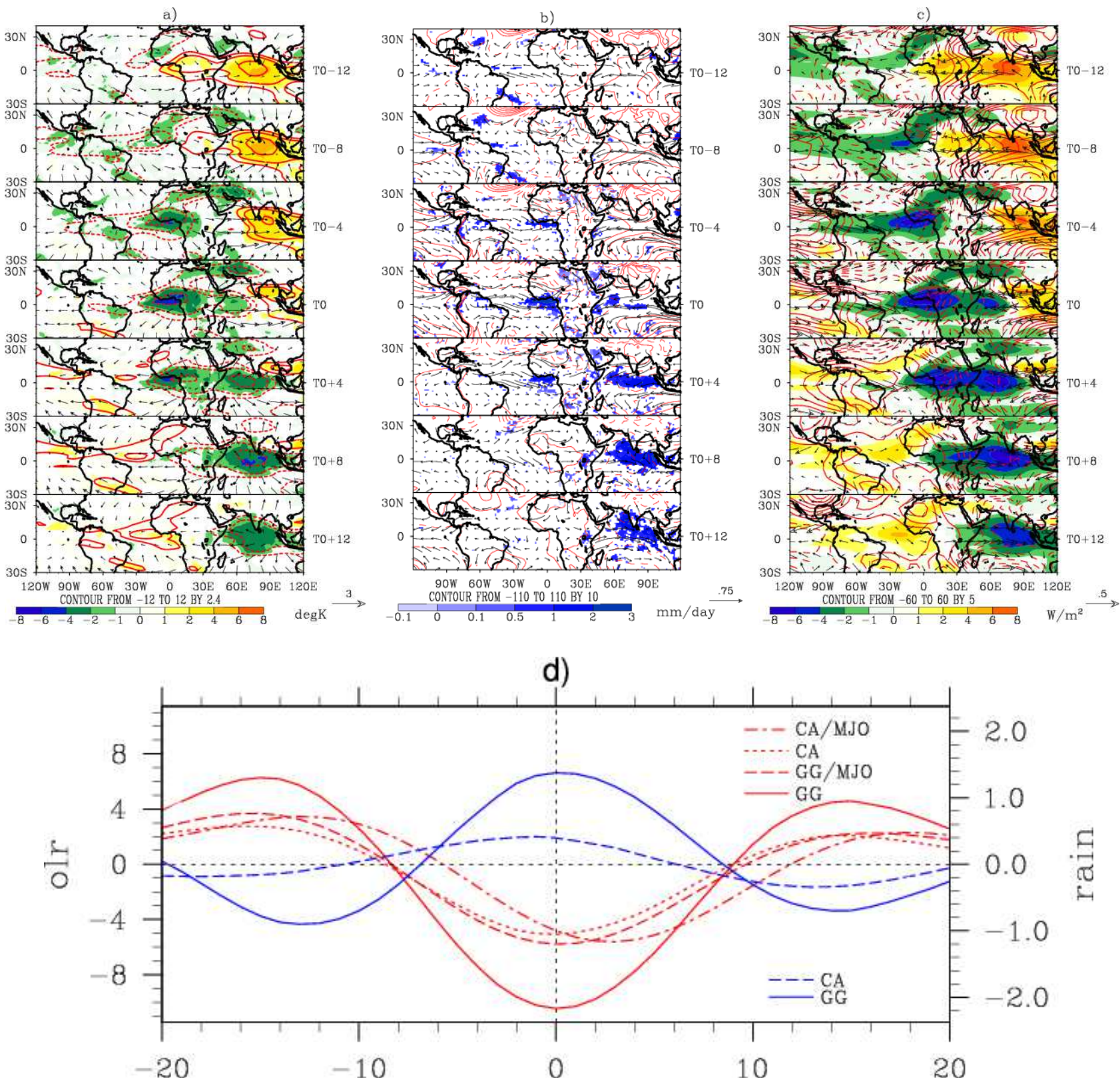

1045

1046

Figure 13

1048

1049

1050

1051

1052 
a)

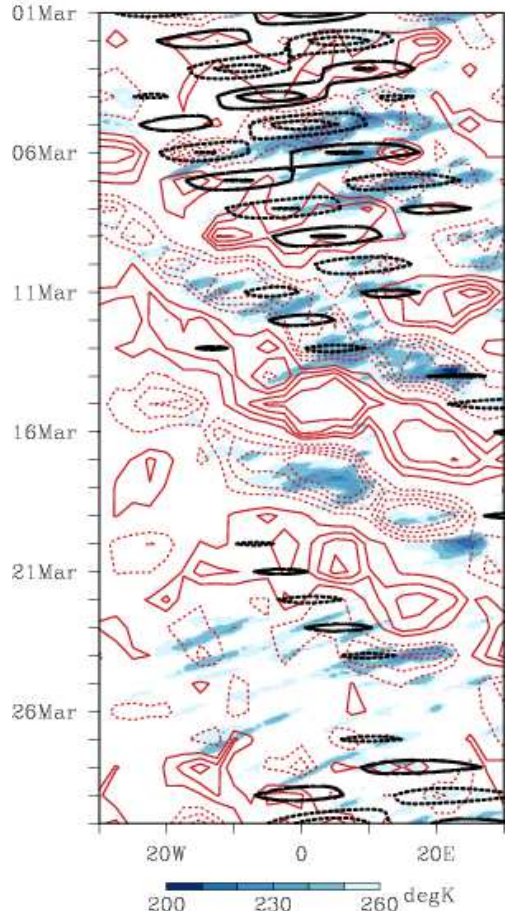

b)

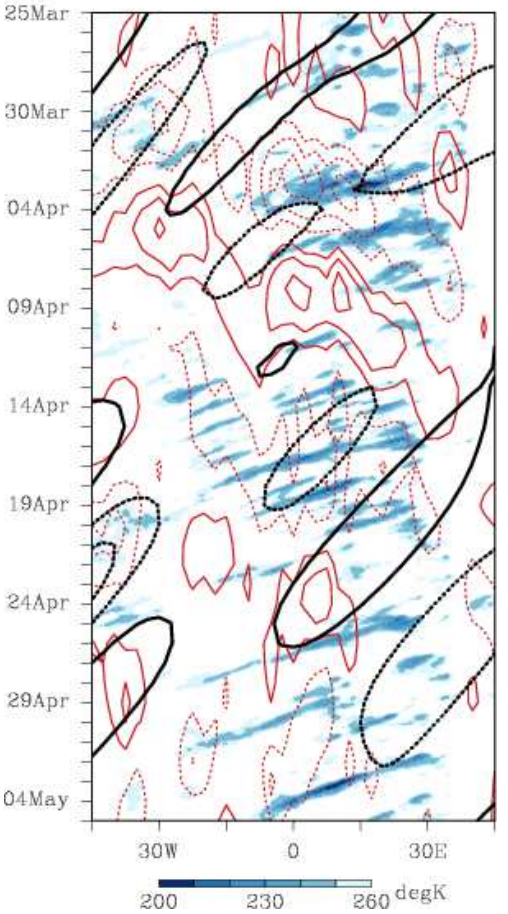

Figure 14 c) 2003 lat $=[2.5 \mathrm{~S}, 7.5 \mathrm{~N}]$

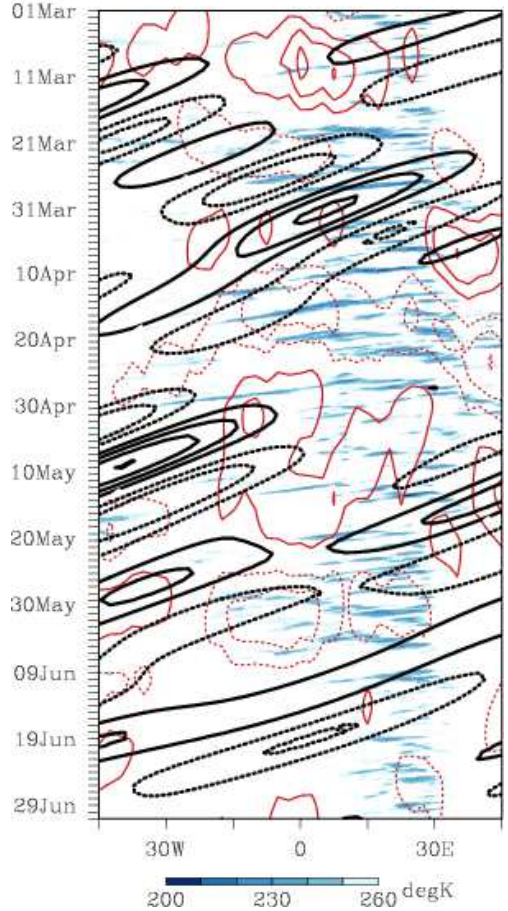

1053

1054

1055
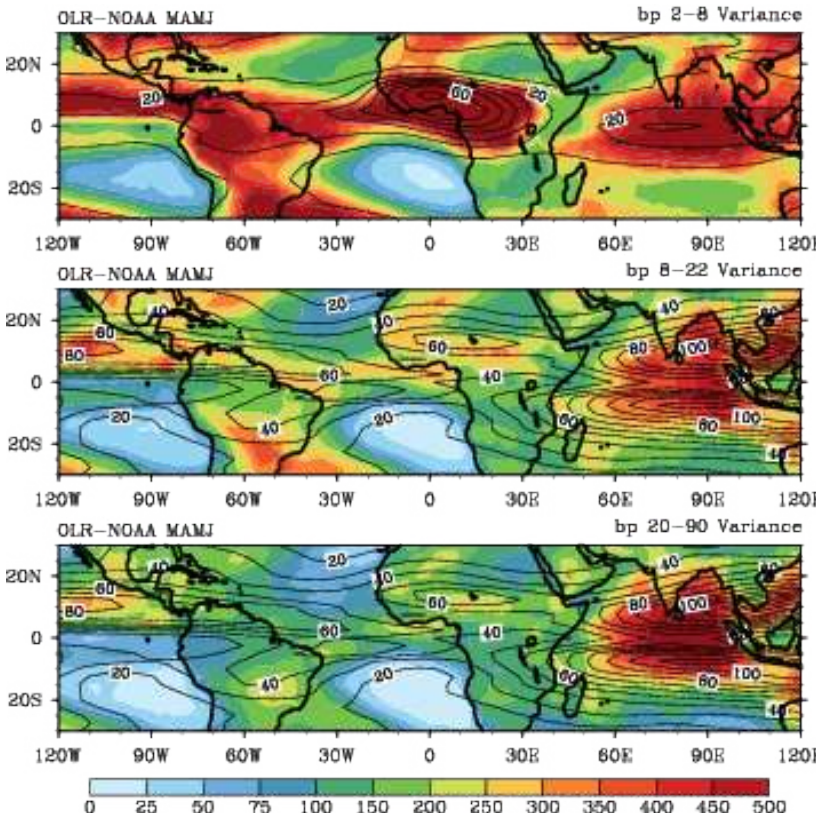
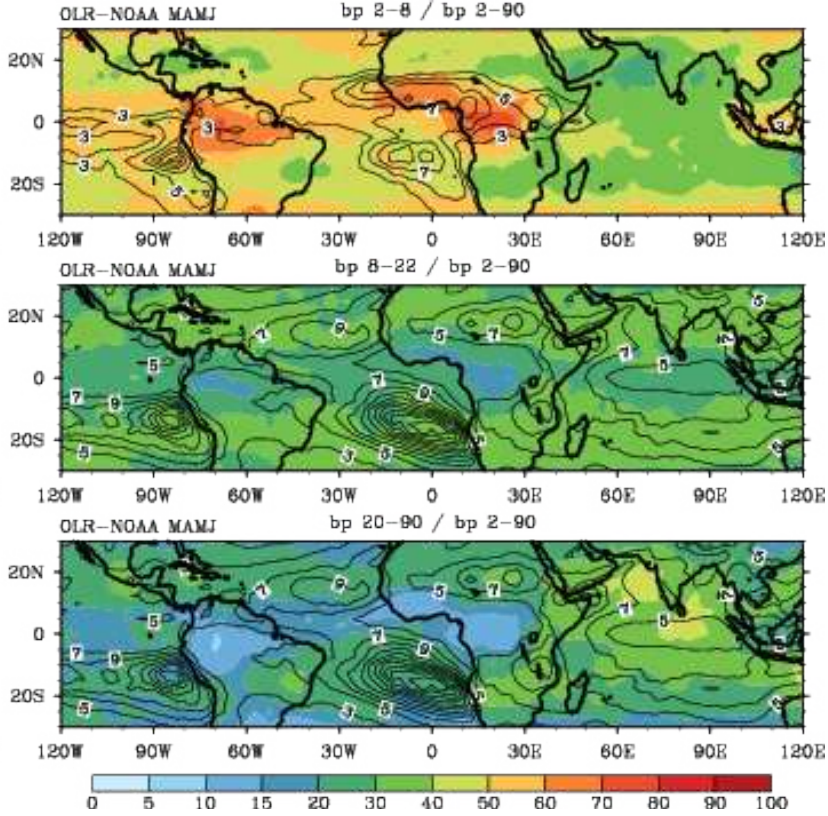

Figure 15 
1058

a)

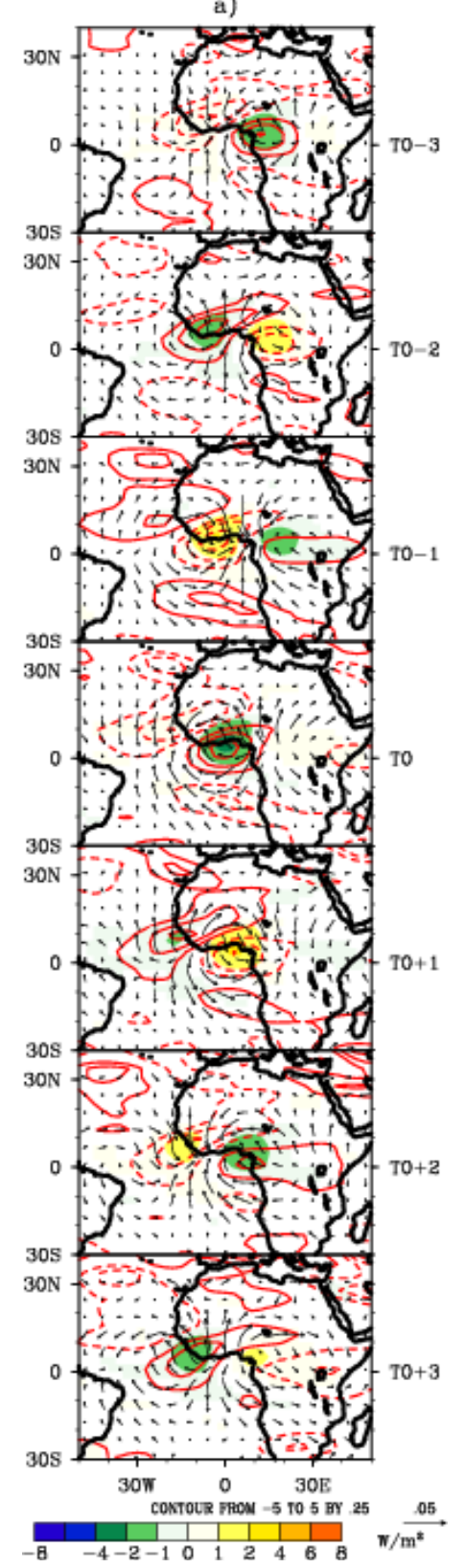

b)
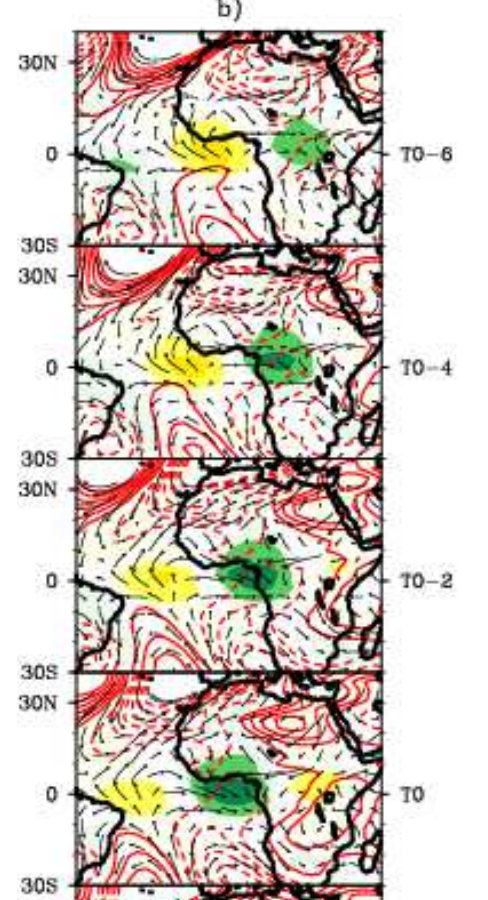

$30 \mathrm{~N}$

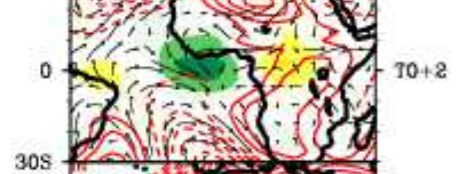

$30 \mathrm{~N}$
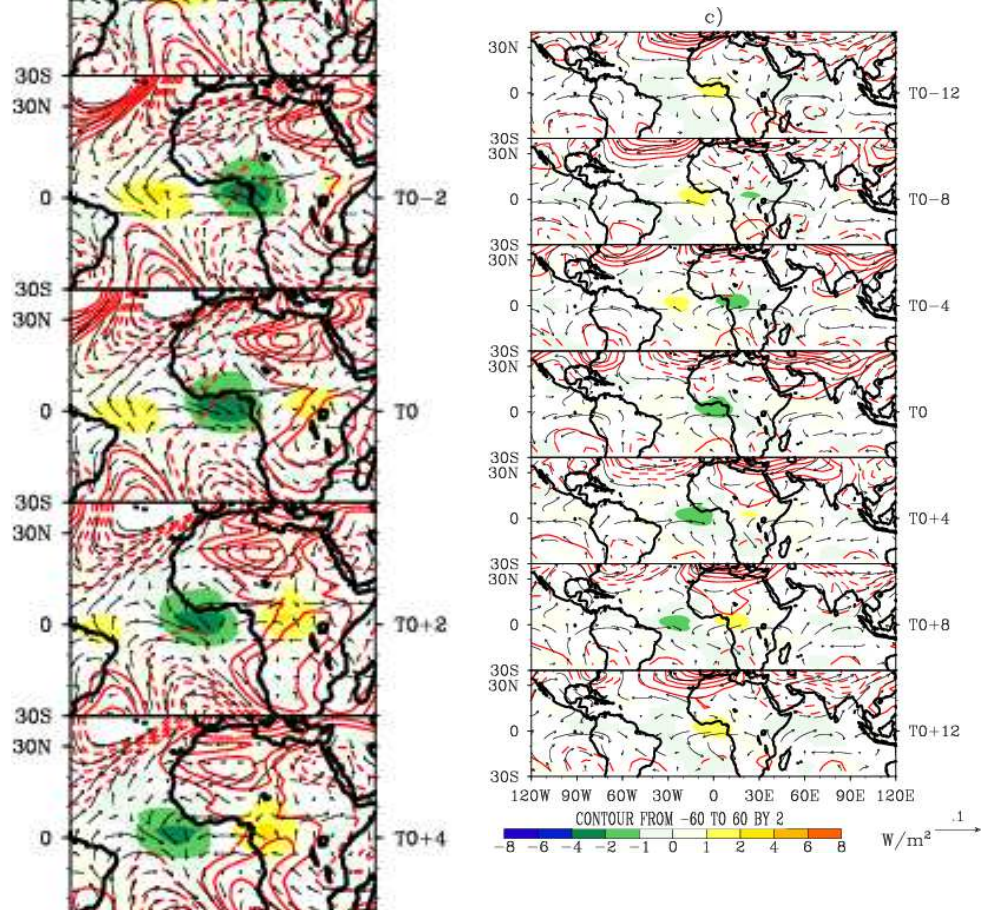

1059

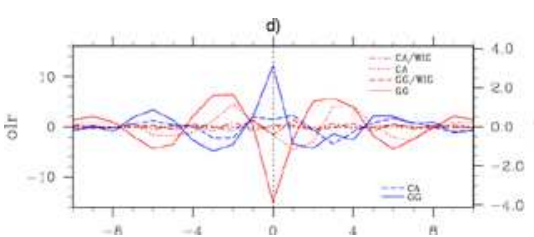

1060

1061

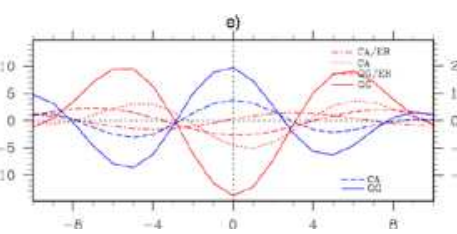

Figure 16
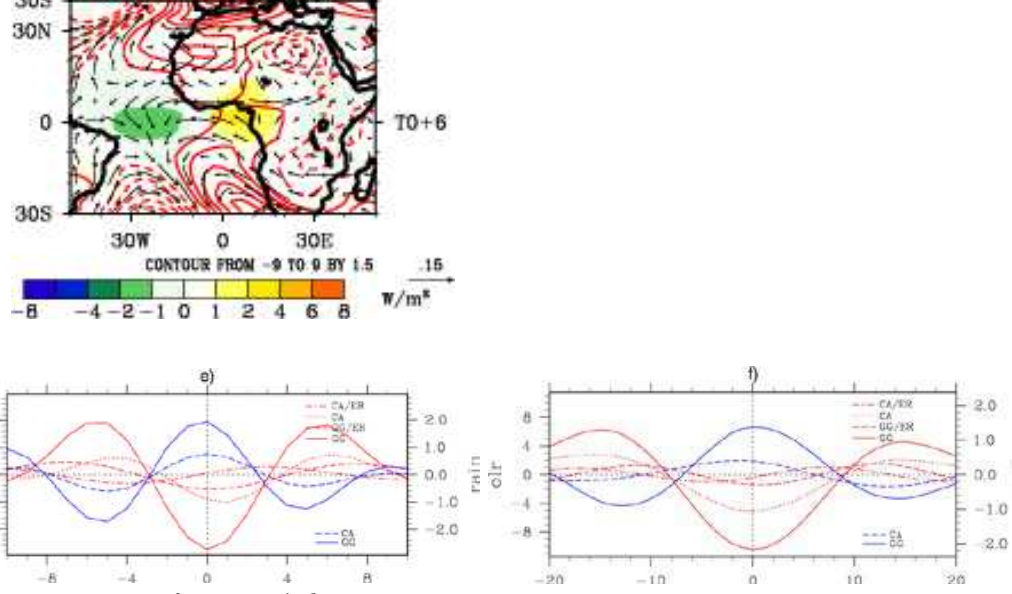